\title{
Tráfico portuario de contenedores: Análisis de causalidad entre los puertos de la Península Ibérica
}

\author{
RAMÓN JiMÉNEZ TORIBIO a , JUAN JOSÉ GARCÍA DEL HOYO a \\ a Universidad de Huelva, Facultad de Ciencias Empresariales y Turismo, Campus de la Merced, \\ Plaza de la Merced, s/n, 21002 Huelva, España.E-mail: toribio@uhu.es, hoyo@uhu.es
}

\begin{abstract}
RESUMEN
El objetivo de este trabajo es estudiar la interacción existente entre los puertos de la Península Ibérica en relación al tráfico de contenedores. Como metodología, se ha empleado el enfoque econométrico propuesto por Toda y Yamamoto (1995).

Se determinó que existe una relación de causalidad unidireccional de Portugal a España, ejerciendo el puerto portugués de Sines una posición de liderazgo sobre los puertos portugueses. Los puertos andaluces presentan una relación de causalidad bidireccional con el conjunto de puertos del resto de España. Por último, a nivel andaluz, se debe destacar la gran importancia del puerto de Algeciras, especialmente en el tráfico de mercancías en tránsito, y la relación de causalidad unidireccional del puerto de Huelva al de Sevilla.

Palabras clave: Tráfico portuario de contenedores, Series temporales, Causalidad, Península Ibérica.
\end{abstract}

\section{Container Port Traffic: Analysis of Causality between the Ports of the Iberian Peninsula}

\begin{abstract}
The objective of this paper is to study the interaction between the ports located in the Iberian Peninsula in relation to container traffic. As a methodology, the econometric approach suggested by Toda and Yamamoto (1995) has been used.

It was determined that there is a unidirectional causal relationship from Portugal to Spain, with the Portuguese port of Sines holding a leading position over the Portuguese ports. The Andalusian ports have a bidirectional causal relationship with ports in the rest of Spain. Finally, at theAndalusian level, it should be noted the great importance of the Algeciras port, especially in the traffic of goods in transit, and the unidirectional causal relationship from the Huelva port to Seville port.

Keywords: Container Port Traffic, Time Series, Causality, Iberian Peninsula.
\end{abstract}

Clasificación JEL: C12, C32, L92

Artículo recibido en julio de 2018 y aceptado en septiembre de 2018

Artículo disponible en versión electrónica en la página www.revista-eea.net, ref. ə-36312 


\section{INTRODUCCIÓN}

El comercio marítimo representa, hoy por hoy, la mayor parte de los flujos comerciales mundiales. La demanda de transporte marítimo constituye una demanda derivada, sujeta a los cambios experimentados por la actividad económica y ello se refleja en las estadísticas de tráfico portuario. En general, el incremento experimentado por los tráficos marítimos en los últimos años siempre ha superado los incrementos en el PIB y ello es debido, fundamentalmente, a las economías de escala del transporte marítimo frente a otros modos de transporte. De hecho, entre 1986 y 2016 el comercio marítimo mundial ha pasado de situarse en torno a 3.500 mill. Tm. anuales a 10.286 mill. Tm. en 2016, un incremento anual medio del 3,7\% (UNCTAD, 2017). Pero esta evolución no ha sido homogénea, de forma que mientras que el petróleo y derivados tan sólo ha crecido a un ritmo del 2,4\% anual, las cargas secas han experimentado un incremento medio del 4,4\%. Pero dentro de éstas, son las mercancías contenerizadas las que han mostrado una evolución más favorable, pasando de apenas 164 mill. Tm. en 1986 a 1.720 mill. Tm. en 2016, con un incremento interanual medio del 8,1\%, que contrasta con los principales graneles sólidos $(4,6 \%)$ y las restantes cargas $(3,1 \%)$. Así, excluyendo los graneles mencionados, del resto de cargas secas las mercancías en contenedores han pasado de representar el 8,3\% en 1980, al 20,4\% en 1998, hasta representar el 42,4\% en 2016. En términos de TEUs, la unidad de medida estándar de contenedores, el volumen transportado ha pasado de 67,3 millones en 1987 a 699,7 millones en 2016, un 940\% de incremento total durante las tres décadas.

Las razones para este incremento son variadas. En primer lugar, las ventajas que presenta la carga contenerizada para favorecer la intermodalidad, unido a la expansión progresiva de las dimensiones medias de los buques portacontenedores auspiciada por la ampliación del Canal de Panamá y la existencia de economías de escala en el transporte por las grandes rutas transoceánicas, aunque algunos estudios indiquen que ya no es posible obtenerlas mediante incrementos adicionales de la dimensión media. Este transporte transoceánico se organiza, fundamentalmente, con un modelo que se denomina "hub and spoke", en la que puertos denominados "hub" reciben contenedores en tránsito de mercancías de puertos secundarios ("local ports" o "gatewayports") -operadas con barcos de dimensión pequeña o media en un tipo de tráfico que se denomina "feeder"- y, en su caso, añadiendo las originadas o destinadas a su "hinterland" o área de influencia. Los puertos locales o de entrada son los encargados de mover las mercancías de su "hinterland". Evidentemente, la mayoría de los puertos combinan ambas funciones, aunque es cierto que el hecho de poder realizar la función de "hubs" depende, en gran medida, de la distancia a las grandes rutas y, sobre todo, del calado disponible en los muelles de las terminales de contenedores. 


\section{EL SISTEMA PORTUARIO EN LA PENÍNSULA IBÉRICA}

El objeto de nuestro trabajo, es analizar la competencia entre los puertos peninsulares en el tráfico de contenedores. En España, existen 28 autoridades portuarias diferentes que gestionan 46 puertos distintos, de las que 23 corresponden a la España continental y las restantes a las ciudades de Ceuta y Melilla y los archipiélagos canario y balear. En Portugal, por su parte, además de los puertos localizados en las Azores y en Madeira, existen en el continente un total de nueve puertos, que se clasifican en principales (Leixões, Aveiro, Lisboa, Setúbal y Sines) y secundarios (Viana do Castelo, Figueira da Foz, Faro y Portimão).

En España, el 75\% de las importaciones y el 58\% de las exportaciones de mercancías se movilizan por vía marítima (MINCOTUR, 2018). Considerando los Puertos de Interés General del Estado, entre 1995 y 2017 las mercancías movilizadas en los puertos casi se han duplicado, pasando de 278 mill. Tm. a 545 mill. Tm., incremento muy superior al experimentado en el mismo periodo por el comercio exterior, que tan sólo ha crecido un 70,5\% en el mismo periodo. Del total de mercancías movilizadas en los puertos españoles en la actualidad, la tercera parte del peso corresponde a mercaderías en contenedores, cuando en 1995 apenas superaban el 14,1\%. Pero de estas mercancías, una proporción que ha oscilado en la última década entre el $50-60 \%$ corresponde al tráfico de tránsito, que ha crecido un $74 \%$ desde 2005 , de forma que en la actualidad representa el $21 \%$ del total de tráficos en el conjunto de los puertos españoles, con 109 mill. Tm. en 2016. De esta forma, el tránsito representa el 53\% de los TEUs movilizados, concentrándose este segmento de negocio en los puertos de Algeciras, Las Palmas, Valencia y Barcelona, que totalizan el 98\% del tráfico, aunque el primero, por sí sólo, moviliza la mitad de los contenedores de transhipment. El comercio exterior propiamente dicho representa el 34,6\% de los TEUs movilizados mientras que el resto corresponde a cabotaje o comercio interior (12,4\%).

En Portugal, por su parte, el movimiento portuario representa tan sólo el $14 \%$ de la suma de las importaciones y exportaciones, dado que gran parte de su comercio exterior es con España y a través de tráfico ferroviario o transporte por carretera (INE, 2018). Desde 1997 el movimiento portuario ha crecido cerca de un 70\%, situándose en la actualidad en 91 mill. Tm., de las cuales, el 14,01\% corresponden a contenedores movidos en los puertos. De hecho, entre 2007 y 2016, el tráfico de mercancías contenerizadas se incrementó en Portugal a un ritmo del 7,7\% interanual, aunque al desagregarlos vemos que frente a un incremento del 10,9\% de los puertos continentales, los puertos insulares tan sólo lo han hecho en un 2,3\%. Pero el incremento de los puertos continentales se debe en gran medida al incremento experimentado por Sines, que entre 2007 y 2016 ha crecido a una tasa acumulativa media del 28,9\% mientras que el resto 
de los puertos tan sólo lo han hecho a un ritmo del 2,3\% anual. Este incremento del tráfico de contenedores en Sines se debe en gran medida al tráfico de tránsito o "transhipment" de contenedores, que representan el 80\% de los TEUs movilizados, con 1,2 millones de TEUs de los 1,5 millones movilizados en 2016.

En conjunto, los puertos de la Península Ibérica de ambos estados representan el 2,3\% del total de los contenedores movilizados a nivel mundial, habiendo crecido a un ritmo medio anual del 5,4\% anual entre 2000 y 2016, por debajo del incremento medio global que se situó en el periodo en 7,2\%. No obstante, comparando con los estados que conformaban la UE-15, la agregación de los puertos de España y Portugal representaban en 2000 el 16,5\% del total y en 2016 se ha situado en el 19,8\%.

Figura 1

Principales rutas oceánicas y regiones portuarias con cruce de rutas

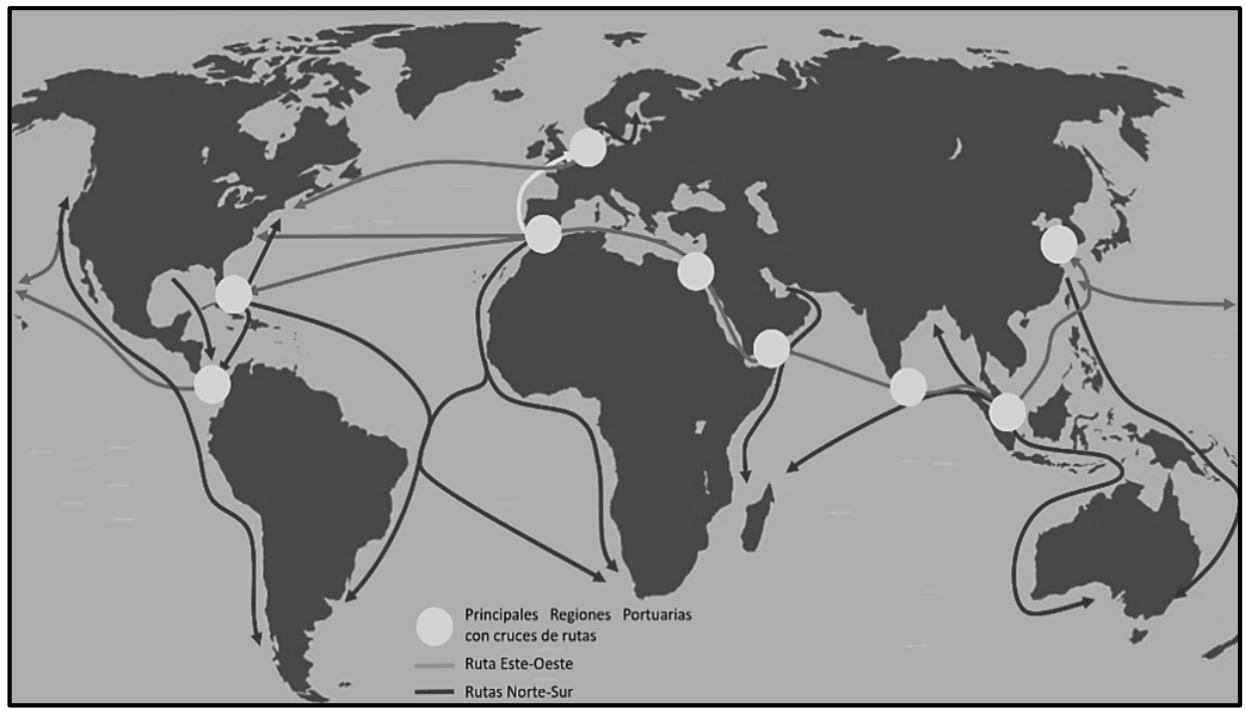

Fuente: Elaboración propia.

Este incremento se debe, en gran medida, a la localización estratégica de la Península Ibérica junto a las grandes rutas oceánicas, la que conecta el extremo oriente con Europa y Norteamérica a través de Singapur, Suez, Gibraltar y Panamá, y de las rutas Norte-Sur (Figura 1). Esta proximidad convierte a algunos de sus puertos (Valencia, Algeciras, Sines y Barcelona) en verdaderos "hubs" o ubicaciones ideales para la carga/descarga de mercancías en tránsito (Merck, 2014). Pero, además, el desarrollo reciente de los corredores ferroviarios europeos, y las mejoras que progresivamente van introduciéndose en las infraestructuras férreas y portuarias para favorecer la intermodalidad, también 
permite que puertos medianos puedan desarrollarse como "gatewayports" (Notteboom, 1997, 2010).

Figura 2

Densidad del tráfico de buques en las costas peninsulares

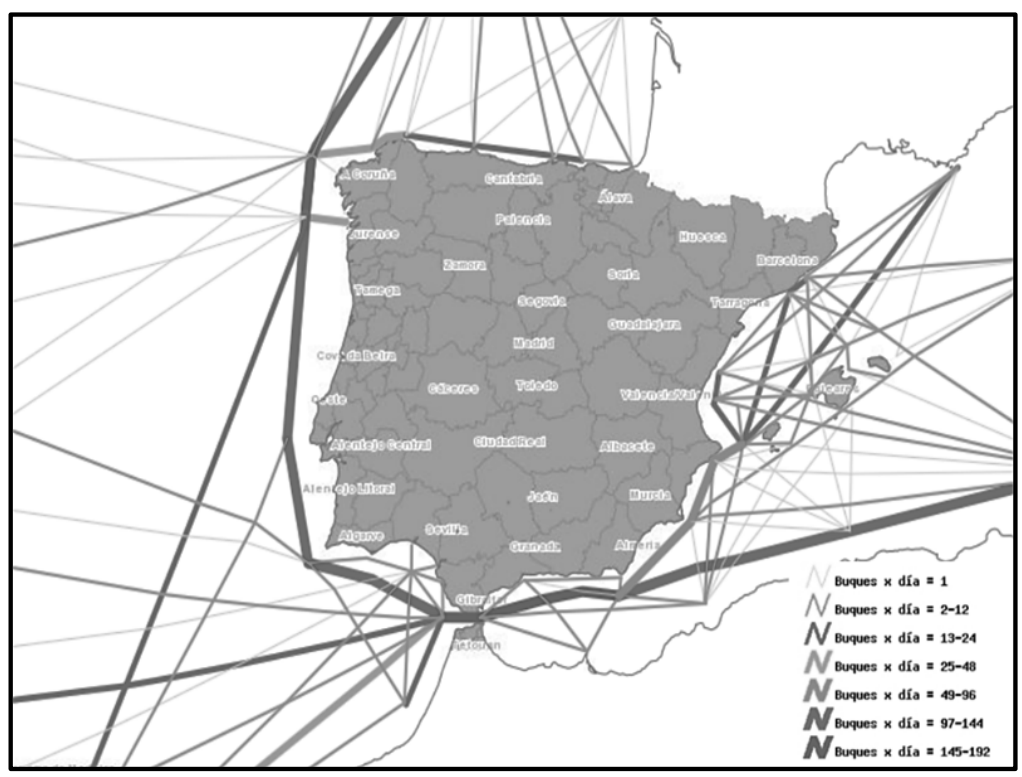

Fuente: Junta de Andalucía, Consejería de Medio Ambiente y Ordenación del Territorio, 2018.

El movimiento de mercancías en tránsito se realiza para reducir los costes e incrementar la tasa de uso de los buques, y existen dos modalidades diferentes: cuando se realiza entre dos buques que cruzan rutas oceánicas de larga distancia ("relé transhipment") y cuando se realiza entre un buque de larga distancia (buque madre) y un buque feeder (hub-and-spoke transhipment), materializándose en puertos localizados en cruces entre rutas oceánicas y rutas de corta distancia. Algunos de los puertos de la Península Ibérica, que permiten el movimiento de grandes buques, se encuentran localizados en el cruce de las rutas Norte-Sur y Este-Oeste, como son Sines o Algeciras, en los que las operaciones de transbordo representan más de 2/3 de los contenedores movilizados, mientras que en otros (Valencia, Barcelona, Málaga) las operaciones de tránsito se sitúan por encima de $1 / 2$ de los contenedores movilizados. Estos cinco puertos concentran prácticamente el $100 \%$ de los contenedores movidos en transhipment y el $60 \%$ de los contenedores de entrada/salida y, en general, compiten fuertemente entre ellos y con otros puertos mediterráneos situados en la ruta Este-Oeste (Tánger-Med, Tarento, Cagliari y Gioia-Tauro). Por su parte, entre los puertos de entrada-salida, el auge del transporte intermodal y el desarrollo de las inversiones en corredores 
ferroviarios hace que para un cargador pueda ser prácticamente indiferente el utilizar puertos más o menos distantes del destino final de la carga (Bergantino, 2002), por lo que el grado de competencia entre los puertos que recepcionan buques feeder se ha incrementado notoriamente en los últimos años. En este sentido, por ejemplo, hay puertos distantes de las grandes rutas que detentan una cuota elevada del tráfico de entrada/salida debido a su "hinterland" (Barcelona, por ejemplo, está a $555 \mathrm{~km}$ de la ruta principal y detenta el $24 \%$ del total del tráfico) mientras que otros muy próximos a la ruta Este-Oeste apenas tienen relevancia debido a las deficientes conexiones intermodales (Almería, por ejemplo, localizada a $48 \mathrm{~km}$ de la ruta y con una cuota de mercado inferior al $1 \%)$, mientras que puertos bien conectados, están realizando ingentes inversiones para incorporarse al negocio del tráfico de contenedores desplazando a otros puertos (Huelva, por ejemplo, cuyo tráfico ha crecido entre 2012 y 2017 un 100,7\%, pasando de 1.700 TEUs a 56.900 TEUs, en detrimento, aparentemente, de los más cercanos de Sevilla y Cádiz) (Figura 2).

En lo que sigue trataremos de analizar la jerarquía e interrelaciones entre los puertos y agregaciones de puertos de la Península Ibérica mediante el análisis econométrico de las series temporales de los movimientos portuarios de mercancías movidas mediante contenedores. Como veremos, los nichos de negocio que hemos descrito en este apartado, van manifestándose a través de los diferentes contrastes de causalidad de Granger que hemos implementado.

\section{DATOS}

Para realizar este trabajo, se han empleado datos mensuales de tráfico de contenedores expresados en TEUs desde enero de 2014 a marzo de 2018. Para recopilar la información necesaria de los puertos situados en España se ha recurrido a las Estadísticas mensuales de Puertos del Estado y para obtener la de los puertos ubicados en Portugal se ha acudido a las estadísticas elaboradas por la Autoridade da Mobilidade e dos Transportes (AMT) y el desaparecido Instituto da Mobilidade e dos Transportes (IMT) (AMT, 2018; IMT, 2018; Puertos del Estado, 2018). El objetivo de estas estadísticas es publicar datos relativos al tráfico portuario de mercancías de los distintos puertos junto con las inversiones efectuadas y las características técnicas de dichos puertos. En la Tabla 1 se presentan los nombres asignados a las variables de este estudio, su definición y la fuente estadística de la información. Igualmente, se han incluido observaciones aclaratorias para algunas de ellas. 
Tabla 1

Definición de las variables y fuente de la información

\begin{tabular}{|c|c|c|c|}
\hline Variable & Definición & Observaciones & Fuente \\
\hline TOT_ESPC & $\begin{array}{l}\text { TEUs totales del total de Puertos de } \\
\text { Interés General de España Continental }\end{array}$ & $\begin{array}{l}\text { Excluye Baleares, Canarias, } \\
\text { Ceuta y Melilla }\end{array}$ & Puertos del Estado \\
\hline TOT_PORTC & $\begin{array}{l}\text { TEUs totales de los puertos de Portugal } \\
\text { Continental }\end{array}$ & $\begin{array}{l}\text { Incluye Viana do Castelo, } \\
\text { Douro y Leixoes, Aveiro, } \\
\text { Figueirada Foz, Lisboa, } \\
\text { Setubal y Sines } \\
\end{array}$ & AMT, IMT \\
\hline REST_PORTC & $\begin{array}{l}\text { TEUs totales de los puertos de Portugal } \\
\text { Continental sin Sines }\end{array}$ & $\begin{array}{l}\text { Incluye Viana do Castelo, } \\
\text { Douro y Leixoes, Aveiro, } \\
\text { Figueira da Foz, Lisboa y } \\
\text { Setubal }\end{array}$ & AMT, IMT \\
\hline SINES & TEUs totales del puerto de Sines & Sin Portimao ni Vila Real & AMT, IMT \\
\hline REST_ESPC & $\begin{array}{l}\text { TEUs totales del Resto de Puertos de } \\
\text { Interés General de España Continental }\end{array}$ & $\begin{array}{l}\text { Excluye Baleares, Canarias, } \\
\text { Ceuta y Melilla y sin } \\
\text { Andalucía }\end{array}$ & Puertos del Estado \\
\hline TOT_AND & $\begin{array}{l}\text { TEUs totales del total de Puertos de } \\
\text { Interés General de Andalucía }\end{array}$ & & Puertos del Estado \\
\hline LEVANTE & $\begin{array}{l}\text { TEUs totales de los puertos del Levante } \\
\text { peninsular }\end{array}$ & De Cartagena a Barcelona & Puertos del Estado \\
\hline NOROESTE & TEUs totales de los puertos de Galicia & & Puertos del Estado \\
\hline NORTE & $\begin{array}{l}\text { TEUs totales de los puertos del } \\
\text { Cantábrico }\end{array}$ & $\begin{array}{l}\text { Cantábrico (Santander, } \\
\text { Asturias, País Vasco) } \\
\end{array}$ & Puertos del Estado \\
\hline ALGECIRAS & $\begin{array}{l}\text { TEUs totales del puerto de Bahía de } \\
\text { Algeciras }\end{array}$ & & Puertos del Estado \\
\hline ALMERIA & TEUs totales del puerto de Almería & & Puertos del Estado \\
\hline CADIZ & $\begin{array}{l}\text { TEUs totales del puerto de Bahía de } \\
\text { Cádiz }\end{array}$ & & Puertos del Estado \\
\hline HUELVA & TEUs totales del puerto de Huelva & & Puertos del Estado \\
\hline MALAGA & TEUs totales del puerto de Málaga & & Puertos del Estado \\
\hline MOTRIL & TEUs totales del puerto de Motril & & Puertos del Estado \\
\hline SEVILLA & TEUs totales del puerto de Sevilla & & Puertos del Estado \\
\hline ALGECIRASST & $\begin{array}{l}\text { TEUs sin tránsito del puerto de Bahía de } \\
\text { Algeciras }\end{array}$ & & Puertos del Estado \\
\hline ALMERIAST & TEUs sin tránsito del puerto de Almería & & Puertos del Estado \\
\hline CADIZST & $\begin{array}{l}\text { TEUs sin tránsito del puerto de Bahía de } \\
\text { Cádiz }\end{array}$ & & Puertos del Estado \\
\hline HUELVAST & TEUs sin tránsito del puerto de Huelva & & Puertos del Estado \\
\hline MALAGAST & TEUs sin tránsito del puerto de Málaga & & Puertos del Estado \\
\hline MOTRILST & TEUs sin tránsito del puerto de Motril & & Puertos del Estado \\
\hline SEVILLAST & TEUs sin tránsito del puerto de Sevilla & & Puertos del Estado \\
\hline ALGECIRASN & $\begin{array}{l}\text { TEUs nacionales del puerto de Bahía de } \\
\text { Algeciras }\end{array}$ & & Puertos del Estado \\
\hline CADIZN & $\begin{array}{l}\text { TEUs nacionales del puerto de Bahía de } \\
\text { Cádiz }\end{array}$ & & Puertos del Estado \\
\hline HUELVAN & TEUs nacionales del puerto de Huelva & & Puertos del Estado \\
\hline MALAGAN & TEUs nacionales del puerto de Málaga & & Puertos del Estado \\
\hline SEVILLAN & TEUs nacionales del puerto de Sevilla & & Puertos del Estado \\
\hline ALGECIRASE & $\begin{array}{l}\text { TEUs de comercio exterior del puerto de } \\
\text { Bahía de Algeciras }\end{array}$ & & Puertos del Estado \\
\hline CADIZE & $\begin{array}{l}\text { TEUs de comercio exterior del puerto de } \\
\text { Bahía de Cádiz }\end{array}$ & & Puertos del Estado \\
\hline
\end{tabular}


Tabla 1 (continuación)

Definición de las variables y fuente de la información

\begin{tabular}{|l|l|c|l|}
\hline \multicolumn{1}{|c|}{ Variable } & \multicolumn{1}{|c|}{ Definición } & Observaciones & \multicolumn{1}{|c|}{ Fuente } \\
\hline HUELVAE & $\begin{array}{l}\text { TEUs de comercio exterior del puerto de } \\
\text { Huelva }\end{array}$ & & Puertos del Estado \\
\hline MALAGAE & $\begin{array}{l}\text { TEUs de comercio exterior del puerto de } \\
\text { Málaga }\end{array}$ & & Puertos del Estado \\
\hline SEVILLAE & $\begin{array}{l}\text { TEUs de comercio exterior del puerto de } \\
\text { Sevilla }\end{array}$ & & Puertos del Estado \\
\hline
\end{tabular}

Fuente: Elaboración propia.

Por otro lado, en la Tabla 2 hemos calculado los principales estadísticos descriptivos de las variables objeto de estudio. En concreto, se han obtenido la media, la desviación típica y los valores mínimo y máximo de cada variable.

Tabla 2

Principales estadísticos descriptivos de las variables utilizadas

\begin{tabular}{|l|c|c|c|c|}
\hline \multicolumn{1}{|c|}{ Variables } & Media & $\begin{array}{c}\text { Desviación } \\
\text { típica }\end{array}$ & Mínimo & Máximo \\
\hline TOT_ESPC & 1.113 .554 & $74.823,29$ & 932.078 & 1.296 .413 \\
\hline TOT_PORTC & $225.402,7$ & $24.957,45$ & 178.336 & 304.356 \\
\hline REST_PORTC & $105.458,1$ & $8.760,91$ & 88.604 & 123.452 \\
\hline SINES & $119.944,6$ & $21.531,66$ & 85.656 & 184.094 \\
\hline REST_ESPC & $707.936,8$ & $66.593,28$ & 562.650 & 871.063 \\
\hline TOT_AND & $405.617,3$ & $35.217,39$ & 305.975 & 460.309 \\
\hline LEVANTE & $627.288,4$ & $63.162,25$ & 493.547 & 785.120 \\
\hline NOROESTE & $23.621,9$ & $2.404,56$ & 18.091 & 31.035 \\
\hline NORTE & $57.027,08$ & $6.218,99$ & 36.976 & 71.956 \\
\hline ALGECIRAS & $377.793,2$ & $34.109,87$ & 279.984 & 433.538 \\
\hline ALMERIA & 527,94 & 134,65 & 229 & 844 \\
\hline CADIZ & $6.428,80$ & $1.138,2$ & 4.221 & 9.144 \\
\hline HUELVA & $1.984,35$ & $2.097,15$ & 201 & 6.872 \\
\hline MALAGA & $6.963,55$ & $7.478,16$ & 2.018 & 39.603 \\
\hline MOTRIL & 117,67 & 138,86 & 0 & 470 \\
\hline SEVILLA & $11.801,8$ & $2.213,15$ & 7.723 & 15.195 \\
\hline ALGECIRASST & $31.651,92$ & $4.748,79$ & 25.639 & 52.146 \\
\hline ALMERIAST & 526,75 & 134,38 & 229 & 844 \\
\hline CADIZST & $6.406,84$ & $1.122,50$ & 4.221 & 9.144 \\
\hline HUELVAST & $1.943,12$ & $2.026,5$ & 201 & 6.718 \\
\hline MALAGAST & $2.955,73$ & 639,04 & 2.018 & 5.587 \\
\hline MOTRILST & 116,41 & 138,25 & 0 & 470 \\
\hline SEVILLAST & $11.801,65$ & $2.212,91$ & 7.723 & 15.195 \\
\hline ALGECIRASN & 273,84 & 236,09 & 8 & 1.231 \\
\hline CADIZN & $3.604,67$ & $1.031,84$ & 2.225 & 5.619 \\
\hline HUELVAN & $1.240,02$ & $1.672,65$ & 40 & 4.982 \\
\hline MALAGAN & $1.272,22$ & 236,53 & 869 & 2.044 \\
\hline SEVILLAN & $11.118,45$ & $1.960,14$ & 7.522 & 14.386 \\
\hline ALGECIRASE & $31.378,08$ & $4.715,94$ & 25.515 & 51.696 \\
\hline
\end{tabular}


Tabla 2 (continuación)

Principales estadísticos descriptivos de las variables utilizadas

\begin{tabular}{|l|c|c|c|c|}
\hline \multicolumn{1}{|c|}{ Variables } & Media & $\begin{array}{c}\text { Desviación } \\
\text { típica }\end{array}$ & Mínimo & Máximo \\
\hline CADIZE & $2.802,18$ & $1.219,96$ & 1.231 & 6.363 \\
\hline HUELVAE & 703,10 & 469,39 & 161 & 1.954 \\
\hline MALAGAE & $1.683,51$ & 593,51 & 839 & 4.051 \\
\hline SEVILLAE & 683,20 & 556,26 & 2 & 2.135 \\
\hline
\end{tabular}

Fuente: Elaboración propia.

\section{METODOLOGÍA}

Con el fin de estudiar la relación de causalidad entre las variables consideradas, en este trabajo se ha utilizado el contraste de causalidad a largo plazo de Toda y Yamamoto (1995). Según Zapata y Rambaldi (1997), esta metodología permite contrastar la causalidad en el sentido de Granger obviando las consecuencias no deseables relacionadas con las propiedades de potencia y tamaño de los contrastes de raíces unitarias y cointegración. En otras palabras, es posible que se pudieran realizar inferencias estadísticas incorrectas sobre la relación de causalidad existente entre las variables simplemente debido a la sensibilidad de los contrastes de raíces unitarias o cointegración a causa de las propiedades de potencia y tamaño anteriormente mencionadas ${ }^{1}$. Mediante este método, se estima un modelo de vector autorregresivo (VAR) con las variables objeto de estudio en niveles que permite reducir los riesgos derivados de la identificación incorrecta del orden de integración de las series temporales y la cointegración entre ellas. Por tanto, la principal virtud de este procedimiento es que no requiere un conocimiento previo del rango de cointegración y, además, es relativamente sencillo de implementar. En particular, el procedimiento de Toda-Yamamoto incrementa el orden correcto del modelo VAR (k) sumándole el máximo orden de integración de las series temporales consideradas $\left(\mathrm{d}_{\max }\right)$. De esta forma, como establece Wolde-Rufael (2005), los estadísticos habituales del contraste de causalidad de Granger tienen la distribución estándar asintótica.

\subsection{Contrastación del orden de integración de las series}

Como paso previo a la implementación del procedimiento de TodaYamamoto, se hace necesario analizar la cuestión de la estacionariedad en las

\footnotetext{
${ }^{1}$ Contrastar la dirección de causalidad se ha realizado generalmente usando los contrastes de Granger (Granger, 1969) o Sims (Sims, 1972). No obstante, estos contrastes se basan en la teoría asintótica, y se ha de tener en cuenta que dicha teoría asintótica únicamente es válida para variables estacionarias (Otero, 1993). Por tanto, si las series consideradas para el análisis de causalidad presentan una raíz unitaria, tales contrastes solamente podrían llevarse a cabo si el modelo VAR se estima con las variables en primeras diferencias, dado que de este modo las variables transformadas serían estacionarias.
} 
series. En este sentido, se ha de determinar el orden de integración de las series, es decir, el número de raíces unitarias existentes en dichas series. Para ello, se ha utilizado el contraste de Dickey-Fuller Aumentado (Dickey y Fuller, 1981). Este contraste se ha realizado tanto sobre las series temporales en niveles como en primeras diferencias. Además, se debe mencionar que el número de retardos (p) a incluir en las regresiones auxiliares del contraste se ha obtenido mediante el criterio de información de Akaike modificado (Ng y Perron, 2001). La inclusión de estos retardos nos permite asegurar que los residuos de las regresiones auxiliares sean ruido blanco.

\subsection{El procedimiento de análisis de la causalidad de Toda-Yamamoto}

Para implementar el método de Toda y Yamamoto (1995), se realiza un contraste modificado de Wald (MWALD) para analizar la causalidad en el sentido de Granger. El contraste MWALD elimina los problemas que surgen en el contraste de causalidad ordinario de Granger cuando no se tiene en cuenta cualquier posible no estacionariedad o cointegración entre las series (Giles, 1997; Zapata y Rambaldi, 1997; Wolde-Rufael 2004, 2005). En este sentido, según Sanjuán López (1998), el contraste de Toda y Yamamoto (1995) consiste en estimar un modelo VAR con las variables en niveles, disminuyéndose los riesgos asociados con la posible identificación incorrecta del orden de integración de las series. Según Wolde-Rufael (2004), para implementar este método, una vez obtenido el orden correcto del modelo VAR $(\mathrm{k})$, se le ha de añadir un número de retardos igual al orden de integración más alto $\left(\mathrm{d}_{\max }\right)$ de las series consideradas. A continuación, se estima un modelo VAR de orden $\mathrm{k}+\mathrm{d}_{\max }$ sin tener en cuenta los coeficientes correspondientes al/a los último/s $\mathrm{d}_{\max }$ retardos (Caporale y Pittis, 1999; Wolde-Rufael, 2004, 2005; Zapata y Rambaldi, 1997). Asimismo, es interesante mencionar que mediante el procedimiento de Toda y Yamamoto (1995) el estadístico habitual del contraste de causalidad tiene la conveniente distribución asintótica pudiéndose realizar inferencias estadísticas válidas. En particular, el estadístico del contraste sigue una distribución asintótica $\chi^{2}$ con $\mathrm{k}$ grados de libertad. Con el fin de ilustrar en mejor medida el procedimiento de Toda y Yamamoto (1995), presentamos como sería el modelo VAR para el caso de dos variables:

$$
\begin{aligned}
& X_{1 t}=\alpha_{0}+\sum_{i=1}^{k} \alpha_{1 i} X_{1, t-i}+\sum_{j=k+1}^{d_{\text {max }}} \alpha_{2 j} X_{1, t-j}+\sum_{i=1}^{k} \varphi_{1 i} X_{2, t-i}+\sum_{j=k+1}^{d_{\text {max }}} \varphi_{2 j} X_{2, t-j}+\varepsilon_{1 t} \\
& X_{2 t}=\beta_{0}+\sum_{i=1}^{k} \beta_{1 i} X_{2, t-i}+\sum_{j=k+1}^{d_{\text {max }}} \beta_{2 j} X_{2, t-j}+\sum_{i=1}^{k} \phi_{1 i} X_{1, t-i}+\sum_{j=k+1}^{d_{\text {max }}} \phi_{2 j} X_{1, t-j}+\varepsilon_{2 t}
\end{aligned}
$$

Para contrastar que $\mathrm{X}_{2}$ no causa en el sentido de Granger a $\mathrm{X}_{1}$, contrastaremos en la ecuación (1) la restricción $\varphi_{1 i}=0 \forall \mathrm{i}$. Del mismo modo, para contrastar que 
$X_{1}$ no causa en el sentido de Granger a $X_{2}$, contrastaremos en la ecuación (1) la restricción $\phi_{1 i}=0 \forall \mathrm{i}$. Para realizar estos contrastes, siguiendo a Dolado y Lütkepohl (1996), se realiza un contraste modificado de Wald basado en estimaciones Mínimo Cuadráticas Ordinarias (MCO) del modelo aumentado expresado en la ecuación (1).

\section{RESULTADOS Y DISCUSIÓN}

Como paso preliminar a la implementación del procedimiento de Toda y Yamamoto (1995) para llevar a cabo el análisis causal, se ha estudiado el orden de integración de las series de tráfico de contenedores consideradas mediante el contraste de Dickey-Fuller Aumentado (Dickey y Fuller, 1981), aplicándose dicho contraste sobre las series en niveles y en primeras diferencias. Considerando los resultados presentados en la Tabla 3, se concluye que las series temporales TOT_PORTC, REST_PORTC, SINES,REST_ESPC, LEVANTE, CADIZ, HUELVA, SEVILLA, CADIZST,HUELVAST,MALAGAST, SEVILLAST, ALGECIRASN,CADIZN, HUELVAN, SEVILLAN, CADIZE,HUELVAE, MALAGAE son I(1) y, por tanto, tienen una raíz unitaria. En cambio, las series temporales TOT_ESPC, TOT_AND, NOROESTE, NORTE,ALGECIRAS, ALMERIA, MALAGA, MOTRIL, ALGECIRASST, ALMERIAST, MOTRILST, MALAGAN, ALGECIRASE, SEVILLAE son I(0), es decir, son estacionarias.

Tabla 3

Contraste de Dickey-Fuller Aumentado

\begin{tabular}{|c|c|c|c|c|c|c|c|}
\hline Variable & Modelo & $p$ & Estadístico T & Variable & Modelo & p & Estadístico $\mathrm{t}$ \\
\hline TOT_ESPC & $\tau_{\tau}$ & 0 & $-5,38^{\star * *}$ & $\triangle T O T \_E S P C$ & $\tau$ & 0 & $-10,40^{* \star *}$ \\
\hline TOT_PORTC & $\tau_{\tau}$ & 2 & $-1,35$ & $\triangle$ TOT_PORTC & $\tau_{\mu}$ & 0 & $-10,91^{\star \star *}$ \\
\hline REST_PORTC & $\tau_{\mu}$ & 4 & $-2,25$ & $\triangle$ REST_PORTC & $\tau$ & 0 & $-11,46^{\star \star \star}$ \\
\hline SINES & $\tau_{\mu}$ & 0 & $-2,55$ & $\triangle$ SINES & $\tau$ & 0 & $-7,79^{\star * \star}$ \\
\hline REST_ESPC & $\bar{\tau} \tau_{\tau}$ & 1 & $-3,15$ & $\triangle \mathrm{REST}$ ESPC & $\bar{\tau}$ & 0 & $-10,77^{\star \star \star}$ \\
\hline TOT_AND & $\tau_{\mu}$ & 2 & $-2,87^{\star}$ & $\triangle T O T \_A N D$ & $\tau$ & 0 & $-9,65^{\star \star \star}$ \\
\hline LEVANTE & $\tau_{\tau}$ & 1 & $-3,06$ & $\triangle$ LEVANTE & $\tau$ & 0 & $-10,75^{\star \star \star}$ \\
\hline NOROESTE & $\bar{\tau}$ & 0 & $-5,52^{\star \star \star}$ & $\triangle$ NOROESTE & $\tau$ & 0 & $-10,26^{\star \star \star}$ \\
\hline NORTE & $\overline{\tau_{\mu}}$ & 0 & $-8,71^{\star \star \star}$ & $\triangle \mathrm{NNORTE}$ & $\tau$ & 0 & $-13,42^{\star \star \star}$ \\
\hline ALGECIRAS & $\tau_{\mu}$ & 0 & $-4,58^{\star \star \star}$ & $\triangle$ ALGECIRAS & $\tau$ & 0 & $-9,09 * \star \star$ \\
\hline ALMERIA & $\tau_{\mu}$ & 0 & $-4,65^{\star \star \star}$ & $\triangle \mathrm{ALMERIA}$ & $\tau$ & 0 & $-8,03^{\star * \star}$ \\
\hline CADIZ & $\tau_{\mu}$ & 5 & $-1,78$ & $\triangle \mathrm{CADIZ}$ & $\tau$ & 1 & $-5,42^{\star \star \star}$ \\
\hline HUELVA & $\tau_{\tau}$ & 2 & $-1,35$ & $\triangle$ HUELVA & $\tau_{\mu}$ & 0 & $-10,91^{\star \star \star}$ \\
\hline MALAGA & $\tau_{\mu}$ & 0 & $-4,61^{\star \star \star}$ & $\triangle \mathrm{MALAGA}$ & $\tau$ & 0 & $-6,70^{\star \star \star}$ \\
\hline MOTRIL & $\tau$ & 2 & $-2,39$ ** & $\Delta$ MOTRIL & $\tau$ & 0 & $-9,73^{\star \star \star}$ \\
\hline SEVILLA & $\tau_{\tau}$ & 5 & $-2,81$ & $\triangle$ SEVILLA & $\tau$ & 0 & $-9,30^{\star \star \star}$ \\
\hline ALGECIRASST & $\tau_{\mu}$ & 2 & $-3,50^{* \star}$ & $\triangle A$ ALGECIRASST & $\tau$ & 0 & $-11,82^{\star \star \star}$ \\
\hline ALMERIAST & $\overline{\tau_{\mu}}$ & 0 & $-4,67^{\star \star \star}$ & $\triangle$ ALMERIAST & $\bar{\tau}$ & 0 & $-8,03^{\star \star \star}$ \\
\hline
\end{tabular}


Tabla 3 (continuación)

Contraste de Dickey-Fuller Aumentado

\begin{tabular}{|c|c|c|c|c|c|c|c|}
\hline Variable & Modelo & p & Estadístico T & Variable & Modelo & p & Estadístico $\mathrm{t}$ \\
\hline CADIZST & $\tau_{\mu}$ & 5 & $-1,79$ & $\triangle \mathrm{CADIZST}$ & $\tau$ & 1 & $-5,54^{\star \star *}$ \\
\hline HUELVAST & $\tau_{\tau}$ & 2 & $-1,45$ & $\triangle$ HUELVAST & $\tau$ & 3 & $-2,95^{\star \star \star}$ \\
\hline MALAGAST & $\tau_{\mu}$ & 4 & $-1,91$ & $\triangle$ MALAGAST & $\tau$ & 0 & $-11,59^{* \star *}$ \\
\hline MOTRILST & $\tau$ & 2 & $-2,41^{\star \star}$ & $\triangle$ MOTRILST & $\tau$ & 0 & $-9,66^{\star \star \star}$ \\
\hline SEVILLAST & $\tau_{\tau}$ & 5 & $-2,81$ & $\triangle$ SEVILLAST & $\tau$ & 0 & $-9,30^{* * *}$ \\
\hline ALGECIRASN & $\tau$ & 3 & $-1,25$ & $\triangle$ ALGECIRASN & $\tau$ & 0 & $-9,48^{\star \star \star}$ \\
\hline CADIZN & $\tau$ & 3 & 0,24 & $\triangle \mathrm{CADIZN}$ & $\tau$ & 1 & $-4,42^{\star \star \star}$ \\
\hline HUELVAN & $\tau_{\tau}$ & 2 & $-1,47$ & $\triangle$ HUELVAN & $\tau$ & 2 & $-3,39 * \star \star$ \\
\hline MALAGAN & $\tau_{\tau}$ & 0 & $-7,48^{\star \star \star}$ & $\triangle$ MALAGAN & $\tau$ & 0 & $-13,00^{* * *}$ \\
\hline SEVILLAN & $\tau_{\tau}$ & 4 & $-2,26$ & $\triangle$ SEVILLAN & $\tau$ & 0 & $-9,19^{* * *}$ \\
\hline ALGECIRASE & $\tau_{\mu}$ & 2 & $-3,48^{\star \star}$ & $\triangle \mathrm{ALGECIRASE}$ & $\tau$ & 0 & $-11,78^{\star \star \star}$ \\
\hline CADIZE & $\tau_{\mu}$ & 3 & $-2,32$ & $\triangle$ CADIZE & $\tau$ & 0 & $-14,27^{\star \star \star}$ \\
\hline HUELVAE & $\tau_{\tau}$ & 1 & $-2,65$ & $\triangle$ HUELVAE & $\tau$ & 1 & $-5,64^{\star \star \star}$ \\
\hline MALAGAE & $\tau$ & 4 & $-0,14$ & $\triangle$ MALAGAE & $\tau$ & 0 & $-10,64^{\star \star \star}$ \\
\hline SEVILLAE & $\tau_{\tau}$ & 0 & $-4,06^{\star *}$ & $\triangle$ SEVILLAE & $\tau$ & 0 & $-7,87^{\star \star \star}$ \\
\hline
\end{tabular}

Nota: Los valores críticos proceden de MacKinnon (1996).

* Estadístico significativo al 10\%. ${ }^{*}$ Estadístico significativo al 5\%. ${ }^{* \star *}$ Estadístico significativo al 1\%.

Modelo: $\tau_{\tau}$ indica que la regresión auxiliar del contraste de Dickey-Fuller Aumentado incluye una constante $y$ una tendencia; $\tau_{\mu}$ indica que sólo incluye una constante; $\tau$ indica que no incluye ninguna componente determinista.

Número máximo de retardos $=6$.

El criterio modificado de Akaike ( $\mathrm{Ng}$ y Perron, 2001) se ha utilizado para la selección del número de retardos de la regresión auxiliar.

Fuente: Elaboración propia.

A continuación, una vez determinado el orden de integración de las series, se ha estimado los modelos VAR con las variables en niveles. Como componente determinista, se ha incluido una constante en los modelos. Para determinar el número de retardos a incluir en los modelos VAR, se ha utilizado en general el criterio de información de Akaike (Lütkepohl, 1993). No obstante, en aquellos casos en los que el modelo VAR seleccionado mediante este criterio ha presentado problemas de autocorrelación serial, se ha modificado el número de retardos a incluir hasta obtener un modelo que fuese válido.

En la Tabla 4 se muestra para cada modelo VAR considerado el número de retardos (k) incluidos y los contrastes multivariantes LM de autocorrelación hasta el orden 1 y hasta el orden 12 (Johansen, 1995). Los resultados indican que los modelos están correctamente especificados para un nivel de significación del 5\%. 
Tabla 4

Contrastes multivariantes de validación de los modelos

\begin{tabular}{|l|c|c|c|}
\hline \multicolumn{1}{|c|}{ Relaciones de variables } & k & $\begin{array}{c}\text { Autocorrelación } \\
\text { (contraste LM) } \\
\text { hasta el orden 1 }\end{array}$ & $\begin{array}{c}\text { Autocorrelación } \\
\text { (contraste } \text { LM) } \\
\text { hasta el orden 12 }\end{array}$ \\
\hline TOT_PORTC/TOT_ESPC & 5 & 1,376739 & 8,592694 \\
\hline SINES/REST_PORTC/TOT_ESPC & 4 & 7,659046 & 11,59209 \\
\hline SINES/REST_PORTC/TOT_AND/REST_ESPC & 4 & 16,5705 & 22,05855 \\
\hline $\begin{array}{l}\text { SINES/REST_PORTC/TOT_AND/LEVANTE/ } \\
\text { NOROESTE/NORTE }\end{array}$ & 4 & 39,25488 & 35,81631 \\
\hline $\begin{array}{l}\text { ALGECIRAS/ALMERIA/CADIZ/HUELVA/MALAGA/ } \\
\text { MOTRIL/SEVILLA }\end{array}$ & 3 & 39,7446 & 61,9726 \\
\hline $\begin{array}{l}\text { ALGECIRASST/ALMERIAST/CADIZST/HUELVAST/MALAGAST/ } \\
\text { MOTRILST/SEVILLAST }\end{array}$ & 3 & 54,72876 & 47,83861 \\
\hline ALGECIRAS/ALMERIA/CADIZ/HUELVA/MALAGA/SEVILLA & 5 & 48,19264 & 49,12267 \\
\hline $\begin{array}{l}\text { ALGECIRASST/ALMERIAST/CADIZST/HUELVAST/ } \\
\text { MALAGAST/SEVILLAST }\end{array}$ & 4 & 41,66558 & 39,42009 \\
\hline MALAGA/CADIZ/HUELVA/SEVILLA/ALGECIRAS & 4 & 21,70235 & 35,31495 \\
\hline MALAGAN/CADIZN/HUELVAN/SEVILLAN/ALGECIRASN & 3 & 27,3697 & 30,63411 \\
\hline MALAGAE/CADIZE/HUELVAE/SEVILLAE/ALGECIRASE & 2 & 22,49989 & 18,68876 \\
\hline
\end{tabular}

Nota: * significativo a un nivel del $5 \%$.

Fuente: Elaboración propia.

Una vez comprobada la validez de los modelos considerados, se ha procedido a realizar los contrastes de causalidad del procedimiento de Toda-Yamamoto. En primer lugar, se ha analizado la dirección de la causalidad entre el conjunto de los puertos españoles (TOT_ESPC) y el conjunto de los puertos portugueses (TOT_PORTC) utilizando el tráfico portuario de mercancías total expresado en TEUs. Como se muestra en la Tabla 5, Portugal causa a España para un nivel de significación del $1 \%$.

Tabla 5

Contraste de causalidad de Toda-Yamamoto para $\mathrm{d}_{\max }=1$ entre TOT_PORTC y TOT_ESPC

\begin{tabular}{|l|c|c|}
\hline $\begin{array}{c}\text { Hipótesis nula (no es causada en sentido } \\
\text { Granger) }\end{array}$ & $\begin{array}{c}\text { Estadísticos } \\
\text { MWALD }\end{array}$ & Dirección de la causalidad \\
\hline $\mathrm{H}_{0}:$ TOT_PORTC vs. TOT_ESPC & 5,910437 & \multirow{2}{*}{ TOT_PORTC $\rightarrow$ TOT_ESPC } \\
\hline $\mathrm{H}_{0}:$ TOT_ESPC vs. TOT_PORTC & $15,9767^{\star \star *}$ & \\
\hline
\end{tabular}

Nota: * Estadístico significativo al 10\%. ${ }^{\star *}$ Estadístico significativo al 5\%. ${ }^{\star \star *}$ Estadístico significativo al $1 \%$. Fuente: Elaboración propia.

Seguidamente, se ha estudiado la dirección de la causalidad entre el puerto de Sines (SINES), el resto de puertos de Portugal (REST_PORTC) y el conjunto de los puertos de España (TOT_ESPC) teniendo en cuenta el tráfico portuario de mercancías total expresado en TEUs. Como se observa en la Tabla 6, Sines causa al Resto de Portugal para un nivel de significación del 5\% y Sines presenta una 
relación de causalidad bidireccional con España (nivel de significación del 10\%). Finalmente, el Resto de Portugal causa a España para un nivel de significación del $1 \%$.

Tabla 6

Contraste de causalidad de Toda-Yamamoto para $d_{\max }=1$ entre SINES, REST_PORTC y TOT_ESPC

\begin{tabular}{|c|c|c|}
\hline $\begin{array}{c}\text { Hipótesis nula (no es causada en sentido } \\
\text { Granger) }\end{array}$ & $\begin{array}{l}\text { Estadísticos } \\
\text { MWALD }\end{array}$ & Dirección de la causalidad \\
\hline $\mathrm{H}_{0}:$ SINES vs. REST_PORTC & 3,569765 & \multirow{2}{*}{ SINES $\rightarrow$ REST_PORTC } \\
\hline $\mathrm{H}_{0}:$ REST_PORTC vS. SINES & $10,73779^{\star \star}$ & \\
\hline $\mathrm{H}_{0}$ : SINES vs. TOT_ESPC & $8,148358^{*}$ & \multirow{2}{*}{ SINES $\leftrightarrow$ TOT_ESPC } \\
\hline $\mathrm{H}_{0}:$ TOT_ESPC vS. SINES & $9,290238^{*}$ & \\
\hline $\mathrm{H}_{0}:$ REST_PORTC vs. TOT_ESPC & 2,795537 & \multirow{2}{*}{ REST_PORTC $\rightarrow$ TOT_ESPC } \\
\hline Ho: TOT_ESPC vs. REST_PORTC & $33,15085^{\star \star \star}$ & \\
\hline
\end{tabular}

Nota: * Estadístico significativo al 10\%. ${ }^{\star *}$ Estadístico significativo al 5\%. *** Estadístico significativo al $1 \%$.

Fuente: Elaboración propia.

A continuación, se ha investigado la dirección de la causalidad entre el puerto de Sines (SINES), el resto de puertos de Portugal (REST_PORTC), el conjunto de los puertos de Andalucía (TOT_AND) y el resto de puertos de España (REST_ESPC) usando el tráfico portuario de mercancías total expresado en TEUs. En la Tabla 7 se muestran los resultados del análisis concluyéndose que Sines causa al resto de puertos de Portugal para un nivel de significación del 10\%. El resto de puertos de Portugal causa al conjunto de puertos andaluces y al Resto de España, ambos a un nivel de significación del 1\%. Finalmente, existe una relación de causalidad bidireccional entre el conjunto de puertos de Andalucía y el resto de puertos de España.

Tabla 7

Contraste de causalidad de Toda-Yamamoto para $d_{\max }=1$ entre SINES, REST_PORTC, TOT_AND y REST_ESPC

\begin{tabular}{|c|c|c|}
\hline $\begin{array}{l}\text { Hipótesis nula (no es causada en sentido } \\
\text { Granger) }\end{array}$ & $\begin{array}{l}\text { Estadísticos } \\
\text { MWALD }\end{array}$ & Dirección de la causalidad \\
\hline $\mathrm{H}_{0}:$ SINES vs. REST_PORTC & 4,376567 & \multirow{2}{*}{ SINES $\rightarrow$ REST_PORTC } \\
\hline $\mathrm{H}_{0}$ : REST_PORTC vs. SINES & $7,981817^{*}$ & \\
\hline HO: SINES vs. por TOT_AND & 3,347483 & \multirow{2}{*}{ No } \\
\hline HO: TOT_AND vs. SINES & 2,381865 & \\
\hline H0: SINES vS. REST_ESPC & 1,449058 & \multirow{2}{*}{ No } \\
\hline HO: REST_ESPC vs. SINES & 7,168443 & \\
\hline HO: REST_PORTC vs. TOT_AND & 3,065974 & \multirow{2}{*}{ REST_PORTC $\rightarrow$ TOT_AND } \\
\hline HO: TOT_AND vs. REST_PORTC & $17,61549^{\star \star \star}$ & \\
\hline H0: REST_PORTC vs. REST_ESPC & 6,238762 & \multirow{2}{*}{ REST_PORTC $\rightarrow$ REST_ESPC } \\
\hline H0: REST_ESPC vS. REST_PORTC & $22,66887^{\star \star \star}$ & \\
\hline H0: TOT_AND vs. REST_ESPC & $9,683461^{\star \star}$ & \multirow{2}{*}{ TOT_AND $\leftrightarrow$ REST_ESPC } \\
\hline HO: REST_ESPC vs. TOT_AND & $13,91454^{\star \star \star}$ & \\
\hline
\end{tabular}

Nota: * Estadístico significativo al 10\%. ** Estadístico significativo al 5\%. ${ }^{* \star \star}$ Estadístico significativo al $1 \%$.

Fuente: Elaboración propia. 
Asimismo, se ha analizado la dirección de la causalidad entre el puerto de Sines (SINES), el resto de puertos de Portugal (REST_PORTC), el conjunto de los puertos de Andalucía (TOT_AND), el conjunto de puertos del Levante (LEVANTE), los puertos gallegos (NOROESTE) y los puertos del Cantábrico (NORTE). Como en los casos anteriores, se ha empleado el tráfico portuario de mercancías total expresado en TEUs. A partir de los resultados mostrados en la Tabla 8, se determina que Sines causa a Galicia y al Cantábrico, ambos a un nivel de significación del 1\%. El resto de puertos de Portugal causa a Galicia y al Levante, ambos a un nivel de significación del 5\%, y también a Andalucía y al Cantábrico, ambos a un nivel de significación del 1\%. Galicia causa a Andalucía a un nivel de significación del 1\%. El Levante causa a Galicia a un nivel de significación del 1\% y al Cantábrico a un nivel de significación del 10\%. El Cantábrico causa a Galicia a un nivel de significación del 5\%. Por último, existe una relación de causalidad bidireccional entre Andalucía y el Levante y, entre Andalucía y el Cantábrico.

\section{Tabla 8}

Contraste de causalidad de Toda-Yamamoto para $d_{\max }=1$ entre SINES, REST_PORTC, TOT_AND, LEVANTE, NOROESTE y NORTE

\begin{tabular}{|c|c|c|}
\hline $\begin{array}{c}\text { Hipótesis nula (no es causada en sentido } \\
\text { Granger) }\end{array}$ & $\begin{array}{l}\text { Estadísticos } \\
\text { MWALD }\end{array}$ & Dirección de la causalidad \\
\hline H0: SINES vs. REST_PORTC & 2,922814 & \multirow{2}{*}{ No } \\
\hline HO: REST_PORTC vS. SINES & 2,702774 & \\
\hline HO: SINES vs. TOT_AND & 2,062422 & \multirow{2}{*}{ No } \\
\hline HO: TOT_AND vs. SINES & 4,457358 & \\
\hline HO: SINES vS. LEVANTE & 3,702632 & \multirow{2}{*}{ No } \\
\hline HO: LEVANTE vs. SINES & 6,548319 & \\
\hline H0: SINES vs. NOROESTE & 1,113702 & \multirow{2}{*}{ SINES $\rightarrow$ NOROESTE } \\
\hline H0: NOROESTE vs. SINES & $25,05676^{\star \star \star}$ & \\
\hline HO: SINES vs. NORTE & 1,92479 & \multirow{2}{*}{ SINES $\rightarrow$ NORTE } \\
\hline H0: NORTE vs. SINES & $16,87375^{\star \star \star}$ & \\
\hline HO: REST_PORTC vs. TOT_AND & 3,370542 & \multirow{2}{*}{ REST_PORTC $\rightarrow$ TOT_AND } \\
\hline HO: TOT_AND vs. REST_PORTC & $13,46709^{\star \star \star}$ & \\
\hline HO: REST_PORTC vs. LEVANTE & 5,13049 & \multirow{2}{*}{ REST_PORTC $\rightarrow$ LEVANTE } \\
\hline HO: LEVANTE vs. REST_PORTC & $12,73634^{\star *}$ & \\
\hline H0: REST_PORTC vs. NOROESTE & 2,659469 & \multirow{2}{*}{ REST_PORTC $\rightarrow$ NOROESTE } \\
\hline H0: NOROESTE vs. REST_PORTC & $11,21987^{\star \star}$ & \\
\hline H0: REST_PORTC vs. NORTE & 6,937448 & \multirow{2}{*}{ REST_PORTC $\rightarrow$ NORTE } \\
\hline H0: NORTE vs. REST_PORTC & $20,86463^{\text {*** }}$ & \\
\hline HO: TOT_AND vs. LEVANTE & $16,09101^{\star \star *}$ & \multirow{2}{*}{ TOT_AND $\leftrightarrow$ LEVANTE } \\
\hline HO: LEVANTE vs. TOT_AND & $11,60588^{\star \star}$ & \\
\hline HO: TOT_AND vs. NOROESTE & $13,58537^{\star \star \star *}$ & \multirow{2}{*}{ TOT_AND $\leftarrow$ NOROESTE } \\
\hline H0: NOROESTE vs. TOT_AND & 5,27791 & \\
\hline HO: TOT_AND vs. NORTE & $12,12692^{\star *}$ & \multirow{2}{*}{ TOT_AND $\leftrightarrow$ NORTE } \\
\hline HO: NORTE vs. TOT_AND & $22,70404^{\star \star * *}$ & \\
\hline H0: LEVANTE vs. NOROESTE & 2,60253 & LEVANTE $\rightarrow$ NOROESTE \\
\hline
\end{tabular}


Tabla 8 (continuación)

Contraste de causalidad de Toda-Yamamoto para $d_{\max }=1$ entre SINES, REST_PORTC TOT_AND, LEVANTE, NOROESTE y NORTE

\begin{tabular}{|c|c|c|}
\hline $\begin{array}{c}\text { Hipótesis nula (no es causada en sentido } \\
\text { Granger) }\end{array}$ & $\begin{array}{l}\text { Estadísticos } \\
\text { MWALD }\end{array}$ & Dirección de la causalidad \\
\hline H0: NOROESTE vs. LEVANTE & $15,63722^{\star \star \star}$ & \\
\hline HO: LEVANTE vs. NORTE & 2,85378 & \multirow{2}{*}{ LEVANTE $\rightarrow$ NORTE } \\
\hline HO: NORTE vs. LEVANTE & $9,067803^{\star}$ & \\
\hline HO: NOROESTE vs. NORTE & $9,621789 * \star$ & \multirow{2}{*}{ NOROESTE $\leftarrow$ NORTE } \\
\hline HO: NORTE vS. NOROESTE & 7,339742 & \\
\hline
\end{tabular}

Nota: * Estadístico significativo al 10\%. ** Estadístico significativo al 5\%. ${ }^{* \star *}$ Estadístico significativo al $1 \%$.

Fuente: Elaboración propia.

Para concluir, nos hemos centrado en los puertos andaluces realizando un estudio de la dirección de causalidad entre ellos. En este sentido, en primer lugar, se ha analizado la dirección de la causalidad entre todos los puertos andaluces (ALGECIRAS, ALMERIA, CADIZ, HUELVA, MALAGA, MOTRIL y SEVILLA), usando el tráfico portuario de mercancías total expresado en TEUs. En la Tabla 9 se presentan los resultados, obteniéndose que Huelva causa a Sevilla a un nivel de significación del 10\%. Almería causa a Málaga a un nivel de significación del 10\%. Sevilla causa a Cádiz a un nivel de significación del 10\% y a Almería a un nivel de significación del 5\%. Finalmente, Cádiz causa a Almería a un nivel de significación del 10\%.

\section{Tabla 9}

Contraste de causalidad de Toda-Yamamoto para $d_{\max }=1$ entre ALGECIRAS, ALMERIA CADIZ, HUELVA, MALAGA, MOTRIL y SEVILLA

\begin{tabular}{|c|c|c|}
\hline $\begin{array}{c}\text { Hipótesis nula (no es causada en sentido } \\
\text { Granger) }\end{array}$ & $\begin{array}{l}\text { Estadísticos } \\
\text { MWALD }\end{array}$ & Dirección de la causalidad \\
\hline H0: ALGECIRAS vs. ALMERIA & 4,389163 & \multirow{2}{*}{ No } \\
\hline H0: ALMERIA vs. ALGECIRAS & 3,344819 & \\
\hline HO: ALGECIRAS vs. CADIZ & 1,226662 & \multirow{2}{*}{ No } \\
\hline H0: CADIZ vs. ALGECIRAS & 5,709057 & \\
\hline H0: ALGECIRAS vs. HUELVA & 5,908459 & \multirow{2}{*}{ No } \\
\hline HO: HUELVA vS. ALGECIRAS & 1,328996 & \\
\hline H0: ALGECIRAS vs. MALAGA & 1,181186 & \multirow{2}{*}{ No } \\
\hline H0: MALAGA vs. ALGECIRAS & 2,505178 & \\
\hline HO: ALGECIRAS vs. MOTRIL & 0,585319 & \multirow{2}{*}{ No } \\
\hline HO: MOTRIL vs. ALGECIRAS & 6,001757 & \\
\hline H0: ALGECIRAS vS. SEVILLA & 5,165923 & \multirow{2}{*}{ No } \\
\hline HO: SEVILLA vS. ALGECIRAS & 1,208773 & \\
\hline HO: ALMERIA vs. CADIZ & $7,684461^{*}$ & \multirow{2}{*}{ ALMERIA $\leftarrow$ CADIZ } \\
\hline HO: CADIZ vs. ALMERIA & 1,595908 & \\
\hline H0: ALMERIA vs. HUELVA & 2,701449 & \multirow{2}{*}{ No } \\
\hline H0: HUELVA vs. ALMERIA & 0,901719 & \\
\hline H0: ALMERIA vs. MALAGA & 4,324629 & ALMERIA $\rightarrow$ MALAGA \\
\hline
\end{tabular}


Tabla 9 (continuación)

Contraste de causalidad de Toda-Yamamoto para $d_{\max }=1$ entre ALGECIRAS, ALMERIA,

CADIZ, HUELVA, MALAGA, MOTRIL y SEVILLA

\begin{tabular}{|c|c|c|}
\hline $\begin{array}{c}\text { Hipótesis nula (no es causada en sentido } \\
\text { Granger) }\end{array}$ & $\begin{array}{l}\text { Estadísticos } \\
\text { MWALD }\end{array}$ & Dirección de la causalidad \\
\hline HO: MALAGA vs. ALMERIA & $6,269641^{*}$ & \\
\hline HO: ALMERIA vs. MOTRIL & 6,024133 & \multirow{2}{*}{ No } \\
\hline HO: MOTRIL vs. ALMERIA & 1,089485 & \\
\hline H0: ALMERIA vs. SEVILLA & $9,168187^{\star *}$ & \multirow{2}{*}{ ALMERIA $\leftarrow$ SEVILLA } \\
\hline HO: SEVILLA vs. ALMERIA & 3,693476 & \\
\hline H0: CADIZ vs. HUELVA & 2,634584 & \multirow{2}{*}{ No } \\
\hline HO: HUELVA vs. CADIZ & 2,847706 & \\
\hline HO: CADIZ vs. MALAGA & 5,996577 & \multirow{2}{*}{ No } \\
\hline HO: MALAGA vs. CADIZ & 1,925798 & \\
\hline HO: CADIZ vs. MOTRIL & 3,860336 & \multirow{2}{*}{ No } \\
\hline HO: MOTRIL vs. CADIZ & 1,539311 & \\
\hline HO: CADIZ vs. SEVILLA & $7,118098^{*}$ & \multirow{2}{*}{ CADIZ $\leftarrow$ SEVILLA } \\
\hline HO: SEVILLA vs. CADIZ & 0,329449 & \\
\hline H0: HUELVA vs. MALAGA & 1,510028 & \multirow{2}{*}{ No } \\
\hline HO: MALAGA vs. HUELVA & 2,176399 & \\
\hline H0: HUELVA vs. MOTRIL & 0,430004 & \multirow{2}{*}{ No } \\
\hline HO: MOTRIL vs. HUELVA & 0,688884 & \\
\hline HO: HUELVA vs. SEVILLA & 2,280799 & \multirow{2}{*}{ HUELVA $\rightarrow$ SEVILLA } \\
\hline HO: SEVILLA vs. HUELVA & $6,987864^{*}$ & \\
\hline HO: MALAGA vs. MOTRIL & 1,647853 & \multirow{2}{*}{ No } \\
\hline H0: MOTRIL vs. MALAGA & 0,499547 & \\
\hline H0: MALAGA vs. SEVILLA & 2,915629 & \multirow{2}{*}{ No } \\
\hline H0: SEVILLA vs. MALAGA & 2,507161 & \\
\hline HO: MOTRIL VS. SEVILLA & 2,074085 & \multirow{2}{*}{ No } \\
\hline HO: SEVILLA vs. MOTRIL & 4,750598 & \\
\hline
\end{tabular}

Nota: * Estadístico significativo al 10\%. ** Estadístico significativo al 5\%. *** Estadístico significativo al $1 \%$.

Fuente: Elaboración propia.

En segundo lugar, se ha investigado la dirección de la causalidad entre todos los puertos andaluces (ALGECIRASST, ALMERIAST, CADIZST, HUELVAST, MALAGAST, MOTRILST y SEVILLAST), usando el tráfico portuario de mercancías sin tránsito expresado en TEUs. Los resultados, que se pueden observar en la Tabla 10, nos permiten concluir que Algeciras causa a Cádiz a un nivel de significación del 1\%. Cádiz causa a Motril a un nivel de significación del 10\%. Huelva causa a Sevilla a un nivel de significación del 10\%. Málaga causa a Huelva a un nivel de significación del 10\%, a Cádiz a un nivel de significación del 5\% y a Motril a un nivel de significación del 5\%. 
Tabla 10

Contraste de causalidad de Toda-Yamamoto para $d_{\max }=1$ entre ALGECIRASST, ALMERIAST, CADIZST, HUELVAST, MALAGAST, MOTRILST y SEVILLAST

\begin{tabular}{|c|c|c|}
\hline $\begin{array}{c}\text { Hipótesis nula (no es causada en sentido } \\
\text { Granger) }\end{array}$ & $\begin{array}{l}\text { Estadísticos } \\
\text { MWALD }\end{array}$ & Dirección de la causalidad \\
\hline H0: ALGECIRASST vs. ALMERIAST & 0,203821 & \multirow{2}{*}{ No } \\
\hline H0: ALMERIAST vs. ALGECIRASST & 1,081797 & \\
\hline HO: ALGECIRASST vs. CADIZST & 1,900959 & \multirow{2}{*}{ ALGECIRASST $\rightarrow$ CADIZST } \\
\hline H0: CADIZST vs. ALGECIRASST & $14,75915^{\star \star \star}$ & \\
\hline H0: ALGECIRASST vs. HUELVAST & 2,981278 & \multirow{2}{*}{ No } \\
\hline H0: HUELVAST vs. ALGECIRASST & 0,954935 & \\
\hline H0: ALGECIRASST vs. MALAGAST & 3,316787 & \multirow{2}{*}{ No } \\
\hline H0: MALAGAST vs. ALGECIRASST & 0,961348 & \\
\hline H0: ALGECIRASST vs. MOTRILST & 0,77992 & \multirow{2}{*}{ No } \\
\hline H0: MOTRILST vs. ALGECIRASST & 0,67905 & \\
\hline H0: ALGECIRASST vs. SEVILLAST & 5,971243 & \multirow{2}{*}{ No } \\
\hline H0: SEVILLAST vS. ALGECIRASST & 0,290052 & \\
\hline H0: ALMERIAST vs. CADIZST & 4,860997 & \multirow{2}{*}{ No } \\
\hline H0: CADIZST vs. ALMERIAST & 1,599459 & \\
\hline H0: ALMERIAST vs. HUELVAST & 0,959579 & \multirow{2}{*}{ No } \\
\hline H0: HUELVAST vs. ALMERIAST & 1,396223 & \\
\hline H0: ALMERIAST vs. MALAGAST & 3,664972 & \multirow{2}{*}{ No } \\
\hline HO: MALAGAST vs. ALMERIAST & 0,003901 & \\
\hline H0: ALMERIAST vs. MOTRILST & 4,31756 & \multirow{2}{*}{ No } \\
\hline H0: MOTRILST vs. ALMERIAST & 0,637416 & \\
\hline H0: ALMERIAST vs. SEVILLAST & 5,545695 & \multirow{2}{*}{ No } \\
\hline H0: SEVILLAST vs. ALMERIAST & 2,267908 & \\
\hline H0: CADIZST vs. HUELVAST & 2,05998 & \multirow{2}{*}{ No } \\
\hline H0: HUELVAST vs. CADIZST & 0,546335 & \\
\hline H0: CADIZST vs. MALAGAST & $9,518944^{\star \star}$ & \multirow{2}{*}{ CADIZST $\leftarrow$ MALAGAST } \\
\hline HO: MALAGAST vs. CADIZST & 3,747487 & \\
\hline HO: CADIZST vs. MOTRILST & 6,152198 & \multirow{2}{*}{ CADIZST $\rightarrow$ MOTRILST } \\
\hline HO: MOTRILST vs. CADIZST & $7,004414^{\star}$ & \\
\hline H0: CADIZST vs. SEVILLAST & 3,642334 & \multirow{2}{*}{ No } \\
\hline H0: SEVILLAST vs. CADIZST & 1,828081 & \\
\hline H0: HUELVAST vs. MALAGAST & $6,979026^{\star}$ & \multirow{2}{*}{ HUELVAST $\leftarrow$ MALAGAST } \\
\hline H0: MALAGAST vs. HUELVAST & 1,428231 & \\
\hline HO: HUELVAST vs. MOTRILST & 0,206253 & \multirow{2}{*}{ No } \\
\hline H0: MOTRILST vs. HUELVAST & 3,005876 & \\
\hline H0: HUELVAST VS. SEVILLAST & 1,367692 & \multirow{2}{*}{ HUELVAST $\rightarrow$ SEVILLAST } \\
\hline H0: SEVILLAST vs. HUELVAST & $6,626965^{\star}$ & \\
\hline H0: MALAGAST vs. MOTRILST & 0,839983 & \multirow{2}{*}{ MALAGAST $\rightarrow$ MOTRILST } \\
\hline H0: MOTRILST vs. MALAGAST & $8,537441^{\star *}$ & \\
\hline H0: MALAGAST vs. SEVILLAST & 1,0934 & \multirow{2}{*}{ No } \\
\hline H0: SEVILLAST vs. MALAGAST & 2,527626 & \\
\hline H0: MOTRILST vs. SEVILLAST & 2,728054 & \multirow{2}{*}{ No } \\
\hline HO: SEVILLAST vs. MOTRILST & 1,235775 & \\
\hline
\end{tabular}

Nota: * Estadístico significativo al 10\%. * Estadístico significativo al 5\%. *** Estadístico significativo al $1 \%$. Fuente: Elaboración propia. 
En tercer lugar, se ha investigado la dirección de la causalidad entre todos los puertos andaluces excluyendo el puerto de Motril (ALGECIRAS, ALMERIA, CADIZ, HUELVA, MALAGA y SEVILLA), que es un puerto cuyo tráfico de mercancías es muy reducido si lo comparamos con los otros puertos andaluces. Para ello, se ha utilizado el tráfico portuario de mercancías total expresado en TEUs. A partir de los resultados de la Tabla 11, se determina que existe una relación de causalidad bidireccional entre Algeciras y Almería, y entre Algeciras y Málaga. Huelva causa a Almería a un nivel de significación del 1\% y a Málaga a un nivel de significación del 5\%. Cádiz causa a Algeciras a un nivel de significación del 5\% y a Málaga a un nivel de significación del 10\%. Sevilla causa a Algeciras y a Cádiz, ambos a un nivel de significación del 10\%, y también a Málaga a un nivel de significación del 1\%. Por último, Málaga causa a Almería a un nivel de significación del 10\%.

Tabla 11

Contraste de causalidad de Toda-Yamamoto para $d_{\max }=1$ entre ALGECIRAS, ALMERIA, CADIZ, HUELVA, MALAGA y SEVILLA

\begin{tabular}{|c|c|c|}
\hline $\begin{array}{c}\text { Hipótesis nula (no es causada en sentido } \\
\text { Granger) }\end{array}$ & $\begin{array}{l}\text { Estadísticos } \\
\text { MWALD }\end{array}$ & Dirección de la causalidad \\
\hline H0: ALGECIRAS vs. ALMERIA & $18,07939^{\star \star \star}$ & \multirow{2}{*}{ ALGECIRAS $\leftrightarrow$ ALMERIA } \\
\hline H0: ALMERIA vs. ALGECIRAS & $29,50673^{\star \star \star}$ & \\
\hline H0: ALGECIRAS vs. CADIZ & $11,93946^{\star \star}$ & \multirow{2}{*}{ ALGECIRAS $\leftarrow$ CADIZ } \\
\hline H0: CADIZ vs. ALGECIRAS & 3,843116 & \\
\hline H0: ALGECIRAS vs. HUELVA & 4,845641 & \multirow{2}{*}{ No } \\
\hline H0: HUELVA vs. ALGECIRAS & 3,22438 & \\
\hline H0: ALGECIRAS vs. MALAGA & $17,64358^{\star \star \star}$ & \multirow{2}{*}{ ALGECIRAS $\leftrightarrow$ MALAGA } \\
\hline H0: MALAGA vS. ALGECIRAS & $11,19414^{\star *}$ & \\
\hline H0: ALGECIRAS vs. SEVILLA & $10,45939^{\star}$ & \multirow{2}{*}{ ALGECIRAS $\leftarrow$ SEVILLA } \\
\hline HO: SEVILLA vs. ALGECIRAS & 1,083355 & \\
\hline H0: ALMERIA vs. CADIZ & 6,053505 & \multirow{2}{*}{ No } \\
\hline HO: CADIZ vs. ALMERIA & 5,659357 & \\
\hline H0: ALMERIA vs. HUELVA & $21,36798^{\star \star \star}$ & \multirow{2}{*}{ ALMERIA $\leftarrow$ HUELVA } \\
\hline H0: HUELVA vs. ALMERIA & 5,982558 & \\
\hline HO: ALMERIA vs. MALAGA & $9,348847^{*}$ & \multirow{2}{*}{ ALMERIA $\leftarrow$ MALAGA } \\
\hline H0: MALAGA vs. ALMERIA & 8,479245 & \\
\hline H0: ALMERIA vS. SEVILLA & 8,953891 & \multirow{2}{*}{ No } \\
\hline HO: SEVILLA vS. ALMERIA & 4,7426 & \\
\hline H0: CADIZ vs. HUELVA & 7,432012 & \multirow{2}{*}{ No } \\
\hline H0: HUELVA vs. CADIZ & 2,540049 & \\
\hline H0: CADIZ vs. MALAGA & 6,52439 & \multirow{2}{*}{ CADIZ $\rightarrow$ MALAGA } \\
\hline H0: MALAGA vs. CADIZ & $10,70517^{*}$ & \\
\hline H0: CADIZ vs. SEVILLA & $9,801545^{*}$ & \multirow{2}{*}{ CADIZ $\leftarrow$ SEVILLA } \\
\hline H0: SEVILLA vs. CADIZ & 1,551456 & \\
\hline H0: HUELVA vs. MALAGA & 4,065362 & \multirow{2}{*}{ HUELVA $\rightarrow$ MALAGA } \\
\hline H0: MALAGA vs. HUELVA & $13,23475^{\star \star}$ & \\
\hline H0: HUELVA vs. SEVILLA & 2,44071 & \multirow{2}{*}{ No } \\
\hline H0: SEVILLA Vs. HUELVA & 2,055095 & \\
\hline
\end{tabular}


Tabla 11 (continuación)

Contraste de causalidad de Toda-Yamamoto para $d_{\max }=1$ entre ALGECIRAS, ALMERIA, CADIZ, HUELVA, MALAGA y SEVILLA

\begin{tabular}{|l|c|c|}
\hline $\begin{array}{c}\text { Hipótesis nula (no es causada en sentido } \\
\text { Granger) }\end{array}$ & $\begin{array}{c}\text { Estadísticos } \\
\text { MWALD }\end{array}$ & Dirección de la causalidad \\
\hline HO: MALAGA vs. SEVILLA & $16,01548^{\star * *}$ & \multirow{2}{*}{ MALAGA $\leftarrow$ SEVILLA } \\
\hline HO: SEVILLA vs. MALAGA & 2,587005 & M \\
\hline
\end{tabular}

Nota: * Estadístico significativo al 10\%. ${ }^{* \star}$ Estadístico significativo al 5\%. ${ }^{* *}$ Estadístico significativo al $1 \%$.

Fuente: Elaboración propia.

En cuarto lugar, se ha llevado a cabo un análisis de la dirección de la causalidad entre todos los puertos andaluces excluyendo el puerto de Motril por la razón expuesta anteriormente (ALGECIRASST, ALMERIAST, CADIZST, HUELVAST, MALAGAST y SEVILLAST), usando el tráfico portuario de mercancías sin tránsito expresado en TEUs. En la Tabla 12 se presentan los resultados obtenidos. Se establece que Algeciras causa a Cádiz y a Sevilla, ambos a un nivel de significación del $10 \%$. Huelva causa a Sevilla a un nivel de significación del 5\%. Málaga causa a Sevilla a un nivel de significación del 5\%. Finalmente, se detecta una relación de causalidad bidireccional entre Sevilla y Almería.

Tabla 12

Contraste de causalidad de Toda-Yamamoto para $d_{\max }=1$ entre ALGECIRASST, ALMERIAST, CADIZST, HUELVAST, MALAGAST y SEVILLAST

\begin{tabular}{|c|c|c|}
\hline $\begin{array}{c}\text { Hipótesis nula (no es causada en sentido } \\
\text { Granger) }\end{array}$ & $\begin{array}{l}\text { Estadísticos } \\
\text { MWALD }\end{array}$ & Dirección de la causalidad \\
\hline H0: ALGECIRASST vs. ALMERIAST & 4,57239 & \multirow{2}{*}{ No } \\
\hline H0: ALMERIAST vs. ALGECIRASST & 1,598445 & \\
\hline H0: ALGECIRASST vs. CADIZST & 1,538377 & \multirow{2}{*}{ ALGECIRASST $\rightarrow$ CADIZST } \\
\hline H0: CADIZST vs. ALGECIRASST & $9,468073^{\star}$ & \\
\hline H0: ALGECIRASST vs. HUELVAST & 5,418351 & \multirow{2}{*}{ No } \\
\hline H0: HUELVAST vs. ALGECIRASST & 2,204681 & \\
\hline H0: ALGECIRASST vs. MALAGAST & 4,188934 & \multirow{2}{*}{ No } \\
\hline H0: MALAGAST vs. ALGECIRASST & 3,518165 & \\
\hline H0: ALGECIRASST vs. SEVILLAST & 2,920206 & \multirow{2}{*}{ ALGECIRASST $\rightarrow$ SEVILLAST } \\
\hline H0: SEVILLAST vs. ALGECIRASST & $8,593144^{*}$ & \\
\hline H0: ALMERIAST vs. CADIZST & 3,230892 & \multirow{2}{*}{ No } \\
\hline H0: CADIZST vs. ALMERIAST & 1,414074 & \\
\hline H0: ALMERIAST vs. HUELVAST & 4,062113 & \multirow{2}{*}{ No } \\
\hline H0: HUELVAST vs. ALMERIAST & 3,809167 & \\
\hline H0: ALMERIAST vs. MALAGAST & 0,950873 & \multirow{2}{*}{ No } \\
\hline H0: MALAGAST vs. ALMERIAST & 2,447624 & \\
\hline H0: ALMERIAST vs. SEVILLAST & $10,94183^{\star \star}$ & \multirow{2}{*}{ ALMERIAST $\leftrightarrow$ SEVILLAST } \\
\hline H0: SEVILLAST vs. ALMERIAST & $9,808124^{\star *}$ & \\
\hline H0: CADIZST vs. HUELVAST & 2,959212 & \multirow{2}{*}{ No } \\
\hline HO: HUELVAST vs. CADIZST & 0,754162 & \\
\hline
\end{tabular}


Tabla 12 (continuación)

Contraste de causalidad de Toda-Yamamoto para $d_{\max }=1$ entre ALGECIRASST, ALMERIAST, CADIZST, HUELVAST, MALAGAST y SEVILLAST

\begin{tabular}{|c|c|c|}
\hline $\begin{array}{c}\text { Hipótesis nula (no es causada en sentido } \\
\text { Granger) }\end{array}$ & $\begin{array}{l}\text { Estadísticos } \\
\text { MWALD }\end{array}$ & Dirección de la causalidad \\
\hline HO: CADIZST vs. MALAGAST & 2,510505 & \multirow{2}{*}{ No } \\
\hline HO: MALAGAST vs. CADIZST & 2,765243 & \\
\hline H0: CADIZST vs. SEVILLAST & 5,98238 & \multirow{2}{*}{ No } \\
\hline H0: SEVILLAST vs. CADIZST & 3,190167 & \\
\hline H0: HUELVAST vs. MALAGAST & 6,138135 & \multirow{2}{*}{ No } \\
\hline HO: MALAGAST vs. HUELVAST & 7,723341 & \\
\hline HO: HUELVAST vs. SEVILLAST & 0,747878 & \multirow{2}{*}{ HUELVAST $\rightarrow$ SEVILLAST } \\
\hline HO: SEVILLAST vs. HUELVAST & $10,03836^{\star *}$ & \\
\hline HO: MALAGAST vs. SEVILLAST & 3,719702 & \multirow{2}{*}{ MALAGAST $\rightarrow$ SEVILLAST } \\
\hline HO: SEVILLAST vS. MALAGAST & $9,516371^{\star *}$ & \\
\hline
\end{tabular}

Nota: * Estadístico significativo al 10\%. ${ }^{* *}$ Estadístico significativo al 5\%. ${ }^{* * *}$ Estadístico significativo al $1 \%$. Fuente: Elaboración propia.

En quinto lugar, se ha estudiado la dirección de la causalidad entre todos los puertos andaluces excluyendo los puertos de Motril y Almería (MALAGA, CADIZ, HUELVA, SEVILLA y ALGECIRAS), que son los que tienen una menor relevancia en la región con respecto al tráfico portuario de mercancías, empleando el tráfico portuario de mercancías total expresado en TEUs. Los resultados se muestran en la Tabla 13, concluyéndose que Sevilla causa a Málaga a un nivel de significación del 10\%. Málaga causa a Algeciras a un nivel de significación del 10\%. Para terminar, Huelva causa a Algeciras a un nivel de significación del 1\%.

Tabla 13

Contraste de causalidad de Toda-Yamamoto para $d_{\max }=1$ entre MALAGA, CADIZ, HUELVA, SEVILLA y ALGECIRAS

\begin{tabular}{|c|c|c|}
\hline $\begin{array}{c}\text { Hipótesis nula (no es causada en sentido } \\
\text { Granger) }\end{array}$ & $\begin{array}{l}\text { Estadísticos } \\
\text { MWALD }\end{array}$ & Dirección de la causalidad \\
\hline H0: MALAGA vs. CADIZ & 3,994576 & \multirow{2}{*}{ No } \\
\hline HO: CADIZ vs. MALAGA & 3,660778 & \\
\hline H0: MALAGA vs. HUELVA & 4,835438 & \multirow{2}{*}{ No } \\
\hline H0: HUELVA vs. MALAGA & 6,263062 & \\
\hline HO: MALAGA vs. SEVILLA & $8,186575^{\star}$ & \multirow{2}{*}{ MALAGA $\leftarrow$ SEVILLA } \\
\hline HO: SEVILLA vs. MALAGA & 1,699936 & \\
\hline HO: MALAGA vs. ALGECIRAS & 5,205493 & \multirow{2}{*}{ MALAGA $\rightarrow$ ALGECIRAS } \\
\hline H0: ALGECIRAS vs. MALAGA & $8,65682^{*}$ & \\
\hline H0: CADIZ vs. HUELVA & 2,774998 & \multirow{2}{*}{ No } \\
\hline HO: HUELVA vs. CADIZ & 2,300058 & \\
\hline HO: CADIZ vs. SEVILLA & 6,682622 & \multirow{2}{*}{ No } \\
\hline H0: SEVILLA vs. CADIZ & 0,559262 & \\
\hline H0: CADIZ vs. ALGECIRAS & 3,426481 & \multirow{2}{*}{ No } \\
\hline H0: ALGECIRAS vs. CADIZ & 4,508617 & \\
\hline
\end{tabular}


Tabla 13 (continuación)

Contraste de causalidad de Toda-Yamamoto para $d_{\max }=1$ entre MALAGA, CADIZ, HUELVA, SEVILLA y ALGECIRAS

\begin{tabular}{|l|c|c|}
\hline $\begin{array}{c}\text { Hipótesis nula (no es causada en sentido } \\
\text { Granger) }\end{array}$ & $\begin{array}{c}\text { Estadísticos } \\
\text { MWALD }\end{array}$ & Dirección de la causalidad \\
\hline H0: HUELVA vs. SEVILLA & 2,588008 & \multirow{2}{*}{ No } \\
\hline H0: SEVILLA vs. HUELVA & 2,459469 & \multirow{2}{*}{ HUELVA $\rightarrow$ ALGECIRAS } \\
\hline H0: HUELVA vs. ALGECIRAS & 0,7801 & \multirow{2}{*}{ No } \\
\hline H0: ALGECIRAS vs. HUELVA & $14,45628^{\star \star \star}$ & \\
\hline H0: SEVILLA vs. ALGECIRAS & 0,486978 & \\
\hline
\end{tabular}

Nota: * Estadístico significativo al 10\%. ** Estadístico significativo al 5\%. ${ }^{\star \star \star ~ E s t a d i ́ s t i c o ~ s i g n i f i c a t i v o ~ a l ~} 1 \%$.

Fuente: Elaboración propia.

En sexto lugar, se ha realizado un análisis de la dirección de la causalidad entre todos los puertos andaluces excluyendo los puertos de Motril y Almería (MALAGAN, CADIZN, HUELVAN, SEVILLAN y ALGECIRASN), como se ha hecho anteriormente, considerando en este caso el tráfico portuario de mercancías nacional expresado en TEUs. A partir de los resultados presentados en la Tabla 14 para un nivel de significación del 1\% en todos estos casos, se determina que Algeciras causa a Málaga y a Huelva. Cádiz causa a Sevilla. Finalmente, Huelva causa a Sevilla.

\section{Tabla 14}

Contraste de causalidad de Toda-Yamamoto para $d_{\max }=1$ entre MALAGAN, CADIZN, HUELVAN, SEVILLAN y ALGECIRASN

\begin{tabular}{|c|c|c|}
\hline $\begin{array}{c}\text { Hipótesis nula (no es causada en sentido } \\
\text { Granger) }\end{array}$ & $\begin{array}{l}\text { Estadísticos } \\
\text { MWALD }\end{array}$ & Dirección de la causalidad \\
\hline H0: MALAGAN vs. CADIZN & 0,420141 & \multirow{2}{*}{ No } \\
\hline H0: CADIZN vs. MALAGAN & 2,079248 & \\
\hline HO: MALAGAN vS. HUELVAN & 4,33762 & \multirow{2}{*}{ No } \\
\hline HO: HUELVAN vs. MALAGAN & 1,636705 & \\
\hline H0: MALAGAN vS. SEVILLAN & 1,851121 & \multirow{2}{*}{ No } \\
\hline H0: SEVILLAN vs. MALAGAN & 5,746968 & \\
\hline HO: MALAGAN vs. ALGECIRASN & $11,89251^{\star \star \star}$ & \multirow{2}{*}{ MALAGAN $\leftarrow$ ALGECIRASN } \\
\hline HO: ALGECIRASN vs. MALAGAN & 0,367009 & \\
\hline HO: CADIZN vs. HUELVAN & 3,538239 & \multirow{2}{*}{ No } \\
\hline H0: HUELVAN vs. CADIZN & 4,287019 & \\
\hline H0: CADIZN vs. SEVILLAN & 5,401639 & \multirow{2}{*}{ CADIZN $\rightarrow$ SEVILLAN } \\
\hline H0: SEVILLAN vS. CADIZN & $12,85898^{\star \star \star}$ & \\
\hline HO: CADIZN vS. ALGECIRASN & 0,936765 & \multirow{2}{*}{ No } \\
\hline H0: ALGECIRASN vs. CADIZN & 3,728165 & \\
\hline H0: HUELVAN vS. SEVILLAN & 1,915164 & \multirow{2}{*}{ HUELVAN $\rightarrow$ SEVILLAN } \\
\hline H0: SEVILLAN vS. HUELVAN & $18,10965^{\star \star \star}$ & \\
\hline HO: HUELVAN vS. ALGECIRASN & $24,931^{\star \star \star}$ & \multirow{2}{*}{ HUELVAN $\leftarrow$ ALGECIRASN } \\
\hline H0: ALGECIRASN vs. HUELVAN & 5,430962 & \\
\hline H0: SEVILLAN vs. ALGECIRASN & 5,537341 & \multirow{2}{*}{ No } \\
\hline H0: ALGECIRASN vs. SEVILLAN & 0,839438 & \\
\hline
\end{tabular}

Nota: * Estadístico significativo al 10\%. ** Estadístico significativo al 5\%. *** Estadístico significativo al $1 \%$.

Fuente: Elaboración propia. 
Para concluir con el análisis de la causalidad entre los puertos andaluces, se ha estudiado la dirección de la causalidad entre todos los puertos andaluces excluyendo los puertos de Motril y Almería (MALAGAE, CADIZE, HUELVAE, SEVILLAE y ALGECIRASE), como se ha hecho en los dos casos anteriores, en este caso teniendo en cuenta el tráfico portuario de mercancías de comercio exterior expresado en TEUs. Los resultados mostrados en la Tabla 15 nos permiten establecer a un nivel de significación del 5\% que, por un lado, Huelva causa a Málaga y, por otro lado, Algeciras causa a Cádiz.

Tabla 15

Contraste de causalidad de Toda-Yamamoto para $d_{\max }=1$ entre MALAGAE, CADIZE, HUELVAE, SEVILLAE y ALGECIRASE

\begin{tabular}{|c|c|c|}
\hline $\begin{array}{c}\text { Hipótesis nula (no es causada en sentido } \\
\text { Granger) }\end{array}$ & $\begin{array}{l}\text { Estadísticos } \\
\text { MWALD }\end{array}$ & Dirección de la causalidad \\
\hline H0: MALAGAE vs. CADIZE & 1,345112 & \multirow{2}{*}{ No } \\
\hline H0: CADIZE vs. MALAGAE & 0,813069 & \\
\hline HO: MALAGAE vs. HUELVAE & $6,63328^{* *}$ & \multirow{2}{*}{ MALAGAE $\leftarrow$ HUELVAE } \\
\hline HO: HUELVAE vs. MALAGAE & 1,132929 & \\
\hline HO: MALAGAE vs. SEVILLAE & 1,094745 & \multirow{2}{*}{ No } \\
\hline HO: SEVILLAE vs. MALAGAE & 0,016163 & \\
\hline H0: MALAGAE vs. ALGECIRASE & 0,14548 & \multirow{2}{*}{ No } \\
\hline H0: ALGECIRASE vs. MALAGAE & 1,949568 & \\
\hline HO: CADIZE vs. HUELVAE & 3,355583 & \multirow{2}{*}{ No } \\
\hline H0: HUELVAE vs. CADIZE & 1,000586 & \\
\hline H0: CADIZE vs. SEVILLAE & 1,157751 & \multirow{2}{*}{ No } \\
\hline HO: SEVILLAE vs. CADIZE & 4,263159 & \\
\hline H0: CADIZE vs. ALGECIRASE & $6,349823^{\star *}$ & \multirow{2}{*}{ CADIZE $\leftarrow$ ALGECIRASE } \\
\hline HO: ALGECIRASE vs. CADIZE & 3,431203 & \\
\hline H0: HUELVAE vs. SEVILLAE & 2,861861 & \multirow{2}{*}{ No } \\
\hline H0: SEVILLAE vs. HUELVAE & 1,347128 & \\
\hline HO: HUELVAE vs. ALGECIRASE & 2,659564 & \multirow{2}{*}{ No } \\
\hline H0: ALGECIRASE vs. HUELVAE & 4,288342 & \\
\hline H0: SEVILLAE vs. ALGECIRASE & 2,968053 & \multirow{2}{*}{ No } \\
\hline H0: ALGECIRASE vs. SEVILLAE & 0,205325 & \\
\hline
\end{tabular}

Nota: * Estadístico significativo al 10\%. ** Estadístico significativo al 5\%. ${ }^{* \star \star}$ Estadístico significativo al $1 \%$. Fuente: Elaboración propia.

\section{CONCLUSIONES}

El objetivo de este trabajo ha sido investigar la relación de causalidad entre los puertos situados en la Península Ibérica en relación al tráfico de contenedores utilizando el procedimiento de Toda y Yamamoto (1995). Para ello, se han estudiado las relaciones de causalidad entre los puertos portugueses y españoles, entre los andaluces y el resto de puertos de la Península Ibérica, y entre el conjunto de puertos andaluces. 
Mediante la aplicación de los contrastes de causalidad de Toda-Yamamoto, en primer lugar, se ha determinado que existe una relación de causalidad unidireccional desde Portugal hacia España, ejerciendo el puerto portugués de Sines una posición de liderazgo sobre el conjunto de los puertos portugueses. Por otro lado, se ha detectado una relación de causalidad unidireccional desde el resto de puertos portugueses hacia el conjunto de puertos de España y una relación de causalidad bidireccional entre Sines y el conjunto de puertos de España. En este sentido, se debe comentar que el gobierno portugués está implementando una estrategia para duplicar el tráfico de contenedores en sus puertos en una década mediante un aumento de la competitividad portuaria entre 2016-2026. Por este motivo, se está realizando un significativo esfuerzo inversor para seguir impulsando el desarrollo y crecimiento de los puertos lusos. Se espera que las mayores inversiones y crecimientos se produzcan en los puertos de Sines, Lisboa y Leixões. Además, con respecto a Sines, habría que añadir que presenta una inmejorable localización estratégica en relación a las rutas marítimas más importantes de tráfico de contenedores; cuenta con una amplia conectividad con otros puertos del continente americano; está mejorando su conectividad ferroviaria lo que está facilitando la firma de acuerdos con otros puertos españoles como el de Sevilla; cuenta con las dos navieras más importantes a nivel mundial, Maersk Line (ML) y Mediterranean Shipping Company (MSC), dado que aunque inicialmente Sines era de MSC, ambas operan gracias a un acuerdo entre ellas; y presenta unas características técnicas que permiten atracar barcos de gran calado (Vasco, 2015). Por todo ello, se justifica el resultado obtenido.

En segundo lugar, el conjunto de puertos de Andalucía presenta una relación de causalidad bidireccional con el conjunto de puertos del resto de España. Asimismo, existe una relación de causalidad unidireccional desde el resto de puertos de Portugal tanto hacia Andalucía como hacia el resto de puertos de España. A nivel de España, es interesante mencionar que los puertos están intentando captar nuevas inversiones al igual que tráficos adicionales de mercancías lo que contribuirá a un incremento de la actividad y, como resultado de ello, a un aumento del empleo. En este contexto, Puertos del Estado (2016) realiza un conjunto de recomendaciones cuya implementación persigue incrementar la competitividad y la eficiencia de los puertos españoles entre las que cabe destacar: favorecer el aumento del número de prestadores de servicios portuarios mediante la supresión de barreras de entrada; desarrollar estudios que comparen los servicios portuarios de España con los de otros puertos de Europa; e implementar un sistema que mida la calidad de los servicios portuarios.

En tercer lugar, empleando un mayor nivel de desagregación de los puertos españoles, se establece que Sines causa a Galicia y al Cantábrico, y el resto de puertos de Portugal causa a Galicia, al Levante, a Andalucía y al Cantábrico. Por tanto, se vuelve a poner de manifiesto el liderazgo de Portugal sobre España en esta materia. Centrándonos en las relaciones de causalidad entre los puertos 
españoles, se determina una relación de causalidad bidireccional entre Andalucía y el Cantábrico, y otra entre Andalucía y el Levante. Por otro lado, se obtienen relaciones de causalidad unidireccional desde el Cantábrico hacia Galicia, desde Galicia hacia Andalucía y desde el Levante hacia Galicia y el Cantábrico.

Finalmente, a nivel andaluz, se ha realizado un análisis utilizando el tráfico portuario de mercancías total, el tráfico portuario de mercancías sin tránsito, el tráfico portuario de mercancías nacional y el tráfico portuario de mercancías de comercio exterior, estando expresados en todos los casos estos tráficos de mercancías en TEUs. Se ha considerado, en primer lugar, todos los puertos andaluces, y posteriormente, nos hemos centrado en los puertos de mayor importancia, excluyendo en primer lugar el puerto de Motril y en segundo lugar el puerto de Almería. Como principales conclusiones, cabe destacar la relación de causalidad unidireccional desde el puerto de Huelva al de Sevilla, lo que pone en evidencia el crecimiento significativo que está experimentando el puerto de Huelva a nivel de tráfico portuario de mercancías frente al de Sevilla gracias, por un lado, a su situación geográfica y sus características técnicas y, por otro lado, al esfuerzo inversor que se está realizando desde la autoridad portuaria para potenciarlo como plataforma logística. Además, se ha de añadir que el puerto de Sevilla está viendo afectada su potencialidad en relación al tráfico portuario de contenedores por la imposibilidad de realizar el dragado del río Guadalquivir como consecuencia del negativo impacto medioambiental que provocaría sobre el estuario y el Parque Nacional de Doñana. Igualmente, cabe mencionar la gran importancia del puerto de Algeciras tanto a nivel de España como de Andalucía, debiéndose destacar su relevancia en el tráfico de mercancías en tránsito. En este sentido, se ha puesto de manifiesto la influencia que el puerto de Algeciras ejerce sobre los puertos de Cádiz, Sevilla y Málaga, aunque en ciertos casos estas relaciones de causalidad son bidireccionales.

Los resultados alcanzados en este trabajo y sus correspondientes conclusiones pueden ser de gran utilidad para la determinación del área de influencia o "hinterland" de cada puerto en relación al tráfico de contenedores y para el estudio de la competencia entre puertos que, como se ha puesto de manifiesto previamente, ha aumentado considerablemente debido al auge del transporte intermodal y al desarrollo de las inversiones en corredores ferroviarios (Bergantino, 2002). En este sentido, el conocimiento del área de influencia o "hinterland" puede resultar de interés a la hora de valorar comercialmente un puerto por los distintos grupos de interés del mercado de transporte marítimo, entre los cuales cabe mencionar a consignatarios, armadores o intermediarios financieros y comerciales. Igualmente, este tipo de investigación puede contribuir al diseño de la política de inversiones de los puertos con el fin de introducir mejoras a nivel logístico que permitan una gestión más eficiente en el ámbito portuario lo que permitirá incrementar el crecimiento comercial y ampliar el área de influencia o "hinterland" de un puerto. 
Las investigaciones futuras podrían estar dirigidas a analizar el área de influencia o "hinterland" no sólo de los puertos situados en la Península Ibérica, sino también a nivel de Europa o incluso a nivel mundial.

Finalmente, se debe comentar que estos resultados se deberían considerar con cautela a causa del tamaño de la muestra. Como afirman Zapata y Rambaldi (1997), el contraste MWALD implementado para analizar la causalidad en el sentido de Granger para desarrollar el método propuesto por Toda y Yamamoto (1995) posee una potencia alta en muestras de un tamaño normal o grande (i.e., más de 50 observaciones). Sin embargo, experimenta una pérdida de potencia en muestras de tamaño pequeño (i.e., igual o menor que 50 observaciones), siendo el tamaño de la muestra considerada en este trabajo ligeramente superior a 50 (51 observaciones).

\section{REFERENCIAS BIBLIOGRÁFICAS}

AMT (2018). "Acompanhamento do Mercado Portuário". Relatorio Mensual. https://www.amt-autoridade.pt.

BERGANTINO, A. S. (2002). "The European Commission approach to Port Policy: some open issues". International Journal of Transport Economics, 29, pp. 337-379.

CAPORALE, G. M. y PITTIS, N. (1999). "Efficient estimation of cointegrating vectors and testing for causality in vector autoregressions". Journal of Economic Surveys, 13(1), pp. 1-35.

DICKEY, D. A. y FULLER, W. A. (1981). "Likelihood ratio statistics for autoregressive time series with a unit root". Econometrica, 49, pp. 1057-1072.

DOLADO, J. J. y LÜTKEPOHL, H. (1996). "Making Wald Tests Work for Cointegrated VAR Systems". Econometric Reviews, 15(4), pp. 369-386.

GILES, D. (1997). "Causality Between the Measured and Underground Economies in New Zealand". Applied Economics Letters, 4, pp. 63-67.

GRANGER, C. (1969). "Investigating Causal Relations by Econometric Models and Cross Spectral Methods". Econometrica, 37, pp. 434-438.

IMT (2018). Movimento de Carga e de Navios nos Portos do Continente. http://www.imtip.pt/sites/IMTT/Portugues/Paginas/IMTHome.aspx.

JOHANSEN, S. (1995). Likelihood-based Inference in Cointegrated Vector Autoregressive Models. Oxford: Oxford UniversityPress.

LUTKEPOHL, H. (1993). Introduction to Multiple Time Series Analysis. Berlin: SpringerVerlag.

MACKINNON, J. G. (1996). "Numerical Distribution Functions for Unit Root and Cointegration Tests". Journal of Applied Econometrics, 11, pp. 601-618.

MERCK, O. (2014). The Competiviness of Global Port-Cities. Paris: OCDE Publishing.

MINCOTUR (2018). Datacomex. Estadísticas del Comercio Exterior Español, http://datacomex.comercio.es/index.htm [Último acceso: 10/5/2018]. 
NOTTEBOOM, T.E. (1997). "Concentration and load centre development in the European container port system". Journal of Transport Geography, 5(2), pp. 99-115.

NOTTEBOOM, T.E. (2010). "Concentration and the formation of multi-port gateway regions in the European container port system: an update". Journal of Transport Geography, 18 (4), pp. 567-583.

NG, S. y PERRON, P. (2001). "Lag length selection and the construction of unit root tests with good size and power". Econometrica, 69(6), pp. 1519-1554.

PUERTOS DEL ESTADO (2016). Informe de competitividad. http://observatorio.puertos.es/DOC_PUBLICOS/INFORME\%20ANUAL\%20DE\%20C OMPETITIVIDAD\%202016.pdf [Último acceso: 23/9/2018].

PUERTOS DEL ESTADO (2018). Estadísticas Mensuales de Tráfico Portuario. http://www.puertos.es/es-es/estadisticas/Paginas/estadistica_mensual.aspx.

OTERO, J. M. (1993). Econometría. Series temporales y predicción. Madrid: Editorial AC.

SAN JUÁN LÓPEZ, A. I. (1998). Integración Espacial de Mercados Agrarios: Una Propuesta Metodológica Aplicada a los Sectores Porcino y Ovino de la UE. Tesis Doctoral, Universidad de Zaragoza.

SIMS, C. (1972). "Money, Income and Causality" en American Economic Review, 62, pp. 540-552.

TODA, H. Y. у YAMAMOTO, T. (1995). "Statistical Inference in Vector Autoregressions with Possibly Integrated Processes". Journal of Econometrics, 66, pp. 225-250.

UNCTAD (2017). Review of Maritime Transport. Nueva York: United Nations Publication.

VASCO, P. (2015). Os potenciais hinterlands dos portos portugueses para cargas contentorizadas, baseado nas acessibilidades rodoviárias. Tesis Doctoral, Universidade do Porto.

WOLDE-RUFAEL, Y. (2004). "Disaggregated industrial energy consumption and GDP: the case of Shanghai, 1952-1999". Energy Economics, 26(1), pp. 69-75.

WOLDE-RUFAEL, Y. (2005). "Energy demand and economic growth: the African experience". Journal of Policy Modeling, 27, pp. 891-903.

ZAPATA, H. O. y RAMBALDI, A. N. (1997). "Monte Carlo evidence on cointegration and causation". Oxford Bulletin of Economics and Statistics, 59, pp. 285-298. 
